\title{
Taming the Basel leverage cycle
}

\author{
Christoph Aymanns ${ }^{\text {a,b,d }}$, Fabio Caccioli ${ }^{c, d, *}$, J. Doyne Farmer ${ }^{\mathrm{a}, \mathrm{b}, \mathrm{e}}$, Vincent W.C. Tan ${ }^{\mathrm{b}}$ \\ a Institute of New Economic Thinking at the Oxford Martin School, University of Oxford, Oxford OX2 6ED, UK \\ ${ }^{\mathrm{b}}$ Mathematical Institute, University of Oxford, Oxford OX1 3LB, UK \\ c Department of Computer Science, University College London, London WC1E 6BT, UK \\ d Systemic Risk Centre, London School of Economics and Political Sciences, London, UK \\ e Santa Fe Institute, Santa Fe, NM 87501, USA
}

\section{A R T I C L E I N F O}

\section{Article history:}

Received 10 December 2014

Received in revised form 14 July 2015

Accepted 18 February 2016

Available online $\mathrm{xxx}$

\section{JEL classification:}

G01

G11

G20

\section{Keywords:}

Financial stability

Capital regulation

Systemic risk

\begin{abstract}
A B S T R A C T
We investigate a simple dynamical model for the systemic risk caused by the use of Value-at-Risk, as mandated by Basel II. The model consists of a bank with a leverage target and an unleveraged fundamentalist investor subject to exogenous noise with clustered volatility. The parameter space has three regions: (i) a stable region, where the system has a fixed point equilibrium; (ii) a locally unstable region, characterized by cycles with chaotic behavior; and (iii) a globally unstable region. A calibration of parameters to data puts the model in region (ii). In this region there is a slowly building price bubble, resembling the period prior to the Global Financial Crisis, followed by a crash resembling the crisis, with a period of approximately 10-15 years. We dub this the Basel leverage cycle. To search for an optimal leverage control policy we propose a criterion based on the ability to minimize risk for a given average leverage. Our model allows us to vary from the procyclical policies of Basel II or III, in which leverage decreases when volatility increases, to countercyclical policies in which leverage increases when volatility increases. We find the best policy depends on the market impact of the bank. Basel II is optimal when the exogenous noise is high, the bank is small and leverage is low; in the opposite limit where the bank is large and leverage is high the optimal policy is closer to constant leverage. In the latter regime systemic risk can be dramatically decreased by lowering the leverage target adjustment speed of the banks. While our model does not show that the financial crisis and the period leading up to it were due to VaR risk management policies, it does suggest that it could have been caused by VaR risk management, and that the housing bubble may have just been the spark that triggered the crisis.
\end{abstract}

(c) 2016 Published by Elsevier B.V.

\section{Introduction}

Borrowing in finance is often called "leverage", which is inspired by the fact that it increases returns, much as a mechanical lever increases force. But leverage also increases risk, which naturally motivates lenders to limit its use. ${ }^{1}$ Because risk is time varying it

\footnotetext{
* Corresponding author at: Department of Computer Science, University College London, London WC1E 6BT, UK. Tel.: +44 (0)20 31087104.

E-mail addresses: christoph.aymanns@gmail.com (C. Aymanns), f.caccioli@ucl.ac.uk (F. Caccioli), Doyne.Farmer@inet.ox.ac.uk (J.D. Farmer), vintwc@gmail.com (V.W.C.Tan).

1 Leverage constraints may arise in a number of ways. If the investor is using collateralized loans it must maintain margin on its collateral. Alternatively, a regulator may impose a risk contingent capital adequacy ratio. A third possibility is that internal risk management considerations may lead the investor to adopt a Value-atRisk constraint (in simple terms Value-at-Risk is a measure of how much the bank could lose with a given small probability). All of these cases effectively impose a risk contingent leverage constraint.
}

is natural to let leverage limits adapt, making them dynamic. But changing leverage has market impact, and as we show here, this can cause systemic effects leading to booms and busts.

It is widely believed that high leverage caused or at least exacerbated the recent financial crisis. The problems are not just excessive risk taking. Because leverage goes up when prices go down, a drop in prices tightens leverage constraints, which may force investors to sell into falling markets, thereby amplifying declines in prices. ${ }^{2}$ This triggers a positive feedback loop in which selling drives prices down, which causes further selling, which further tightens leverage constraints, etc. Similarly, positive news about prices causes a decline in perceived risk, which loosens leverage constraints,

\footnotetext{
${ }^{2}$ In principle, distressed banks can reduce their leverage in two ways: they can raise more capital or sell assets. In practice most banks tend to do the latter, as documented in Adrian and Shin (2008).
} 


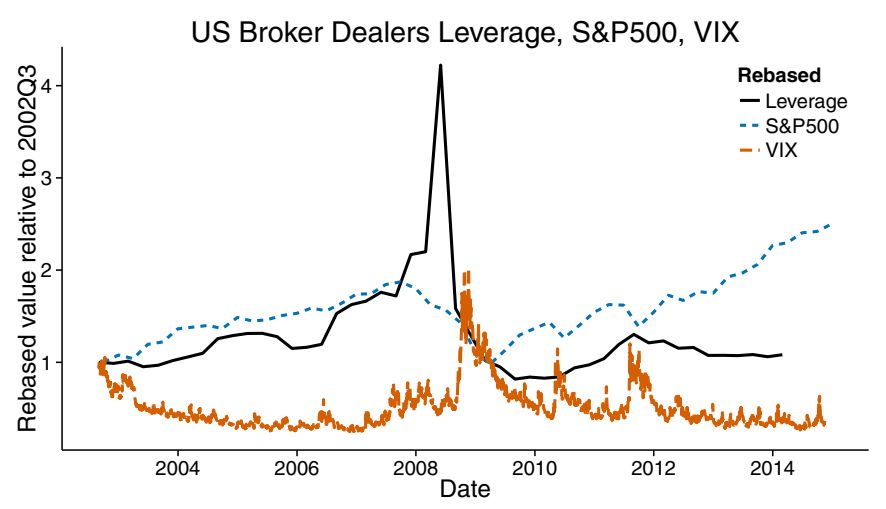

Fig. 1. The leverage of US Broker-Dealers (solid black line) compared to the S\&P500 index (dashed blue line) and the VIX S\&P500 (red dash-dotted line). Data is quarterly. ${ }^{4}$ (For interpretation of the references to color in this figure legend, the reader is referred to the web version of this article.)

driving prices up. Dynamics of this general type were termed the leverage cycle by Geanakoplos. ${ }^{3}$

The crisis has focused a great deal of attention on leverage cycles. During the period leading up to the financial crisis perceived volatility declined consistently over several years while asset prices and leverage of financial institutions consistently increased. We refer to this period as "Great Financial Moderation" in analogy to the Great Moderation - a period of low business cycle volatility starting in the 1980 s coined by Ben Bernanke.

This is illustrated in Fig. 1, where we show the behavior of the VIX index, the S\&P500 index and the leverage of US security broker dealers. These trends came to a sudden halt in 2008 when leverage came plummeting down, dropping by almost a factor of two in the span of a quarter, while volatility rose dramatically and prices decreased.

The cause of these events has been a matter of great debate, with many theories being offered. Prior to the crisis, displaying remarkable prescience, several authors worried that the Basel II regulations were dangerous because of their potential to cause or at least exacerbate financial instability, ${ }^{5}$ and since the crisis several models have been developed that support this conclusion. ${ }^{6}$ Our paper adds to this literature by refining an earlier model by Aymanns and Farmer (2015) and making it more quantitative. The model endogenously generates a leverage cycle whose

${ }^{3}$ Minsky (1992) was the first to describe leverage cycles in qualitative terms. An early model discussing the destabilizing effects of leverage was presented by Gennotte and Leland (1990); see also work by Shleifer and Vishny (1992) on fire sales debt capacity over the business cycle; a literature review is given in Shleifer and Vishny (2011). The first quantitative model of the leverage cycle per se is due to Geanakoplos (1997, 2003); See also Fostel and Geanakoplos (2008) and Geanakoplos (2010). Brunnermeier and Pedersen (2008) investigate the destabilizing feedback between funding liquidity and market liquidity, and the destabilizing effects of margin are discussed in Gorton and Metrick (2010). Other mechanisms for leverage cycles include Aikman et al. (2015), de Nicolo et al. (2012) and Gennaioli et al. (2012).

4 It should be noted that US security broker dealers are a somewhat extreme example of leveraged financial institutions and are not representative for the behavior of commercial banks. Here we use their example to illustrate the stark correlation between leverage, volatility and asset prices in an anecdotal way. A more nuanced evaluation can be found in (Adrian et al., 2010). The data on US Security Broker Dealer Leverage, (defined as Assets/(Assets-Liabilities), is from US Federal Reserve Flow of Funds Data Package F.128 available at www.federalreserve.gov/datadownload/.

${ }^{5}$ Authors who presaged the potential of Basel II to amplify financial instability include Danielsson et al. (2001), Van den Heuvel (2002), Danielsson et al. (2004) and Estrella (2004).

${ }^{6}$ See for example Adrian and Shin (2008), Shin (2010), Zigrand et al. (2010), Thurner et al. (2010), Adrian et al. (2012), Tasca and Battiston (2012), He and Krishnamurthy (2012), Adrian and Boyarchenko (2012, 2013), Poledna et al. (2013), Adrian and Shin (2014), and Brummitt et al. (2014). properties roughly match the Great Financial Moderation and subsequent crisis, including the correct timescale, based on simple assumptions.

Our model has only two representative investors, a bank and a fundamentalist fund, whose portfolios consist of a risky asset and cash. The fund buys the risky asset when it is undervalued and sells it when it is overvalued. The bank follows a leverage target based on a Value-at-Risk criterion, as recommended by Basel $\mathrm{II}^{7}$. Risk is estimated by a moving average of historical volatility. The risk appetite of the bank is controlled by a parameter $\alpha$. When $\alpha$ is small, leverage, prices and volatility converge to a stable fixed point equilibrium. But at a critical value of $\alpha$ the equilibrium becomes unstable and the dynamics suddenly become chaotic, making a finite amplitude oscillation. When this happens the behavior resembles that shown in Fig. 1: prices and leverage slowly rise while volatility falls, as they did during the Great Financial Moderation, and then a crisis occurs in which prices and leverage abruptly drop while volatility spikes upward. This cycle repeats itself indefinitely, but with chaotic variations from period to period.

Following Aymanns and Farmer we call this the Basel leverage cycle. Perhaps the most surprising aspect is that it persists even in the limit where there is no exogenous noise. A simple calibration based on reasonable values of the parameters yields a period of oscillation of roughly 10-15 years. This suggests that VaR as mandated by Basel II was sufficient to cause the Great Financial Moderation and the subsequent crash, and the collapse of the housing bubble might have just been one of several possible triggers for the crisis. The Aymanns and Farmer model was inspired by the empirical findings of Adrian and Shin (2008), who pointed out that investors such as investment banks are actively procyclical, i.e. they lower leverage targets when prices fall and raise them when prices rise. They argued that this is due to regulatory risk management based on Value-at-Risk. In the following we use their terminology a bit differently, and refer to a procyclical leverage control policy as one for which banks are required to reduce their target leverage when volatility increases, and are allowed to increase it when volatility decreases. Since volatility and returns are negatively correlated (Black, 1976; Christie, 1982; Nelson, 1991; Engle and Ng, 1993), leverage procyclicality induces a positive feedback between the demand for an asset and its return, which is what we wish to capture here.

We refer to the opposite case in which leverage and volatility go up and down together as a countercyclical leverage control policy. The leverage control policy used in our model contains a parameter that makes it possible to move continuously between these two extremes. Not surprisingly, countercyclical leverage control policies can also generate instabilities. The challenge for policy makers is to find a policy that avoids the Scylla and Charybdis of excessively procyclical behavior on one side or excessively countercyclical behavior on the other. ${ }^{8}$

A key enhancement of the Aymanns and Farmer model that we make here is that the exogenous noise affecting the risky asset has clustered volatility, i.e. the amplitude of the noise varies in time. This allows us to study the tradeoff between micro and

\footnotetext{
7 An external regulator is not necessary - prudent risk managers may choose to use VaR on their own, while failing to take the systemic consequences into account

${ }^{8}$ It has to be stressed that the concept of cyclicality we refer to in this paper is with respect to risk, not with respect to the behavior of macroeconomic indicators For example, Drehmann and Gambacorta (2012) provide counterfactual simulations showing how leverage control policies that are countercyclical with respect to the difference between the credit-to-GDP ratio and its long-run average can help making the economy more stable. The focus of our paper, however, is on the circumstances in which leverage control can cause financial instability, and how to make an effective tradeoff between systemic vs. individual risk.
} 
macroprudential regulation. In the limit where the bank is small the optimal policy is Basel II. As the bank becomes larger, however, there is the potential for the system to endogenously generate systemic risk, and things become more complicated. The best policy depends on the parameters of the system. We also make several others changes to their model, making the noise trader a fundamentalist, and restructuring the model so that it has a continuum limit and so the parameters are easier to interpret, allowing a better match to real data.

We formulate a criterion for an optimal leverage control policy. Equity capital is widely regarded by practitioners as expensive (see however Admati and Hellwig (2014)). We assume that this leads banks to choose policies that maximize leverage at a given level of overall risk. This is desirable because this means that the capital of the financial system is put to full use in providing credit to the real economy. In fact, for reasons of convenience it is more feasible for us to minimize risk at a given leverage, which is essentially equivalent. We measure risk in terms of realized shortfall, i.e. the average of large losses to the financial system as a whole.

One of the main results of this paper is that the optimal policy depends critically on three parameters: (1) the average leverage used by the bank, (2) the relative size of the bank and the fundamentalist and (3) the amplitude of the exogenous noise. A procyclical leverage control policy such as that of Basel II is optimal when the exogenous noise is high and the volatility is strongly clustered, the bank is small and leverage is low; in the opposite limit where these conditions are not met the optimal policy is closer to constant leverage.

We explicitly assume bounded rationality, using assumptions that are simple, plausible and supported by empirical evidence. In Section 6.2 we argue that this is justifiable for several reasons, including the manifest failure of rationality in the period leading up to the crisis of 2008.

This paper is organized as follows: In Section 2 we describe the model in broad terms, leaving some of the details to Appendix A. In Section 3 we discuss how the parameters affect the behavior of the model and present an overview of its behavior. In Section 4 we perform a stability analysis. In Section 5 we present our criterion for an optimal leverage policy and study how the best policy depends on the circumstances. Finally in Section 6 we give a summary and defend our choice of bounded rationality.

\section{A simple model of leverage cycles}

\subsection{Sketch of the model}

We consider a financial system composed of a leveraged investor, which we call the bank, an unleveraged fund investor, which we call the fund, and a passive outside lender that provides credit as required by the bank. The bank and the fund make a choice between investing in a risky asset whose price is determined endogenously vs. a risk free asset with fixed price, which we will call cash. The market clearing price of the risky asset is determined by the excess demand of the fund and the bank. Fig. 2 shows a diagrammatic representation of the model.

We focus on risk management by assuming the bank holds the relative weight of the risky asset and cash fixed. The bank's risk management consists of two components. First, the bank estimates the future volatility of its investment in the risky asset through an exponential moving average of historical returns. Second, the bank uses the estimated volatility to set its desired leverage. If the bank is below its desired leverage, it will borrow more and use the additional funds to expand its balance sheet; if it is above its desired leverage, it will liquidate part of its investments and pay back part of its debt. We call this leverage targeting.

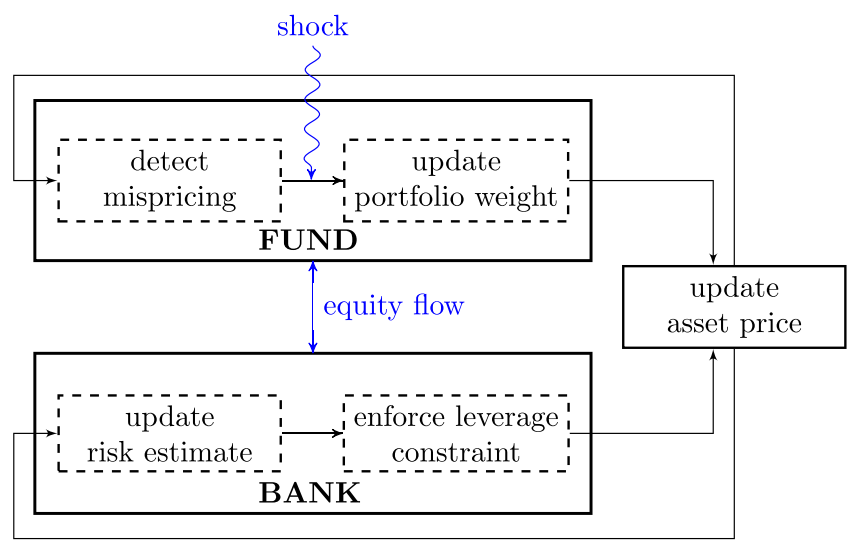

Fig. 2. Diagrammatic representation of the model: The bank and the fund interact through price formation. The bank's demand for the risky asset depends on its estimated risk based on historical volatility and on its capital requirement. The demand of the fund consists of a mean reverting component that tends to push the price towards its fundamental value; in addition there is a random exogenous shock to the fund's demand that has clustered volatility. Price adjustments affect the bank's estimation of risk and the mean reverting behavior of the fund. The cash flow consistency in the model is enforced by equity flowing between the bank and the fund in equal amounts. The driver of the endogenous dynamics is the feedback loop between price changes, volatility and demand for the risky asset.

The fund is a proxy for the rest of the financial system. Leverage targeting creates inherently unstable dynamics, as it implies buying into rising markets and selling into falling markets. Thus, it is necessary to have at least one other investor to stabilize the system. The fund plays this role, holding the risky asset when it is underpriced and shorting it when it is overpriced. The fund's investment decisions are perturbed by exogenous random shocks with clustered volatility, based on a GARCH model, reflecting information flow or decision processes outside the scope of the model.

The bank tries to maintain a constant equity target. This is consistent with the empirical observation that the equity of commercial and investment banks is roughly constant over time; see Adrian and Shin (2008). In order to conserve cash flow in our model, dividends paid out by the bank when the equity exceeds the target are invested in the fund, while new capital invested in the bank when the equity is below the target is withdrawn from the fund. This prevents wealth from accumulating with either the bank or the fund and makes the asymptotic dynamics stationary.

\subsection{Leverage regulation}

The most important ingredient of our model is the fact that the bank has a capital requirement. The leverage ratio ${ }^{9}$ is defined as

$\lambda(t)=\frac{\text { Total Assets }}{\text { Equity }}$,

and the capital requirement policy implies a constraint of the form $\lambda(t) \leq \bar{\lambda}(t)$, i.e. the bank is allowed a maximum leverage $\bar{\lambda}(t)$. For convenience, we assume the bank always targets its maximum allowed leverage $\bar{\lambda}(t) .{ }^{10}$ This depends on the bank's estimate of the volatility of the risky asset, i.e. $\bar{\lambda}(t)=F\left(\sigma^{2}(t)\right)$, where $F$ is a function

\footnotetext{
9 We use this definition of leverage in analogy to the Tier 1 regulatory leverage ratio (Tier 1 capital over bank total assets).

10 A cap on leverage is equivalent to a minimum capital buffer. Conditional on the leverage constraint the return on equity of the bank is maximized if $\lambda(t)=\bar{\lambda}(t)$ (see for instance Shin (2010)). In reality banks usually keep more capital than required by regulation in order to reduce the cost of recapitalization or portfolio adjustments associated with violation of the minimal capital requirement. Using this perspective, Peura and Keppo (2006) explain the pattern of capital buffers observed in a sample of US commercial banks. Our results remain valid even if we assume that banks hold more capital than required by the regulator. We only require that the resulting
} 
of the bank's perceived risk at time $t$ denoted as $\sigma^{2}(t)$. Although nothing we do here depends on this, to gain intuition it is useful to compute the function $F$ under the special case of a Value-atRisk constraint with normally distributed log returns. In this case the bank's target leverage is given by (see for example Corsi et al. (2013)):

$\bar{\lambda}(t)=F_{\mathrm{VaR}}\left(\sigma^{2}(t)\right)=\frac{1}{\sigma(t) \Phi^{-1}(a)} \propto \frac{1}{\sigma(t)}$,

where $\Phi$ is the cumulative distribution of the standard normal, $a$ is the VaR quantile, and $\sigma$ is the volatility of the risky asset. Under this specification the bank increases its leverage when the volatility of the risky asset diminishes and decreases its leverage in the opposite case. Motivated by Adrian and Shin (2014), we classify leverage policies as follows:

Definition 1. A leverage policy $F\left(\sigma^{2}(t)\right)$ is procyclical if $d F / d \sigma^{2}<0$ and countercyclical if $d F / d \sigma^{2}>0 .{ }^{11}$

A class of leverage control policies that allows us to interpolate between procyclical and countercyclial leverage control policies is given by

$\bar{\lambda}(t)=F_{\left(\alpha, \sigma_{0}^{2}, b\right)}(\sigma(t)):=\alpha\left(\sigma^{2}(t)+\sigma_{0}^{2}\right)^{b}$,

where $\alpha>0, \sigma_{0}^{2}>0$ and $b \in[-0.5,0.5]$. We refer to $\alpha$ as the bank's riskiness. The larger $\alpha$ the larger the bank's target leverage for a given level of perceived risk $\sigma^{2}(t) .{ }^{12}$ We illustrate the range of leverage control policies in Fig. $3 .^{13}$

The parameter $b$ is called the cyclicality parameter, due to the fact that $F_{\left(\alpha, \sigma_{0}^{2}, b\right)}$ is procyclical for $b<0$ and countercyclical for $b>0$ (see Definition 1 ). For procyclical policies the leverage is inversely related to risk, i.e. leverage is low when risk is high and vice versa. For countercyclical policies the opposite is true; when risk is high leverage is also high, see Fig. 3. It is important to note that our definition of policy cyclicality does not refer to macroeconomic measures such the credit-to-GDP ratio or asset prices. Instead, it is defined solely by the bank's response to changes in perceived risk. In this sense, the countercyclical policies proposed in this model differ from the countercyclical capital buffer proposed by the Bank of England, which keys off the credit-to-GDP ratio (see FPC (2014)).

\subsection{Asset price dynamics}

The bank's target leverage $\bar{\lambda}(t)$ at time $t$ defines a target portfolio value $\bar{A}_{B}(t)=\bar{\lambda}(t) E_{B}(t)$, where $E_{\mathrm{B}}(t)$ is the equity of the bank. The difference between the target portfolio and the current portfolio then determines the change of the balance sheet $\Delta B(t)$ required for the bank to achieve its target leverage:

bank capital buffer responds to changes in perceived risk in a well defined way, as changes in the capital buffer are more important than its level.

11 This definition could be generalized for any risk measure; we use the standard deviation $\sigma$ for simplicity.

12 Note that under standard Value-at-Risk the bank's leverage depends on the variance of its entire portfolio which in our model includes non-risky cash holdings. Usually the portfolio variance is computed as the inner product of the covariance matrix with the portfolio weights. In our case this implies that the portfolio variance is simply $\sigma^{2}(t)$ scaled by the bank's investment weight in the risky asset $w_{\mathrm{B}}$. However, since we take $w_{\mathrm{B}}$ constant throughout, the resulting risk rescaling factor can be absorbed into $\alpha$ without loss of generality. Therefore, we make $F_{\left(\alpha, \sigma_{0}^{2}, b\right)}$ only a function of $\sigma(t)$.

13 The additional scaling parameter $\sigma_{0}^{2}>0$ is included to bound the cyclical variation in target leverage when perceived risk is very low, giving an upper bound in the procyclical case $b<0$ and a lower bound in the countercyclical case $b>0$.

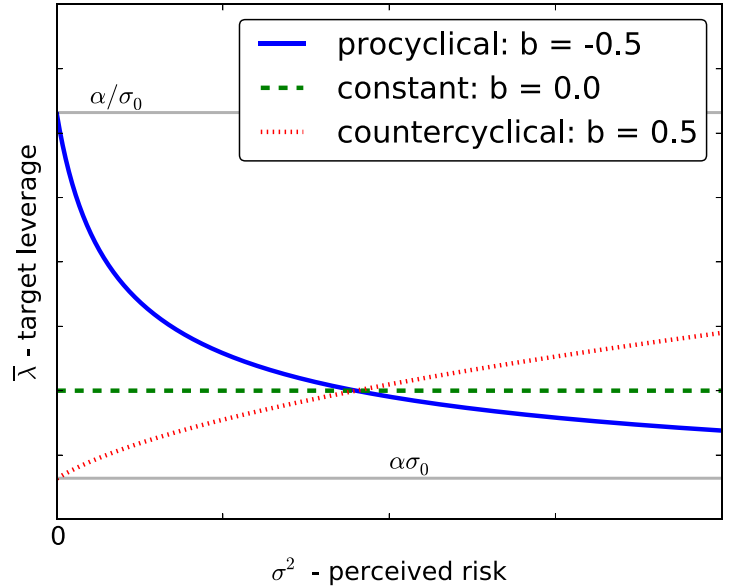

Fig. 3. Illustration of target leverage as a function of perceived risk based on Eq. (2) with $\sigma_{0}^{2}>0$. Continuous blue line: procyclical policy with $b=-0.5$. Dashed green line: constant leverage policy with $b=0$. Dotted red line: countercyclical policy with $b=0.5$. Continuous grey lines illustrate the role of $\sigma_{0}$, which acts as a upper bound on leverage when $b<0$ and a lower bound when $b>0$. (For interpretation of the references to color in this figure legend, the reader is referred to the web version of this article.)

- If $\Delta B(t)>0$, the bank will borrow $\Delta B(t)$ and invest this amount into the risky and the risk free asset according the bank's portfolio weights.

- If $\Delta B(t)<0$, the bank will liquidate part of its portfolio and pay back $\Delta B(t)$ of its liabilities.

The evolution of the fund's portfolio weight in the risky asset depends on the asset's price relative to a constant fundamental value $\mu$, and also on random innovations. The fund investor therefore combines two economic mechanisms: (1) The price of the risky asset is weakly anchored by the performance of unmodeled macroeconomic conditions, which we assume are effectively constant over the length of one run of our model. To achieve this the fund invests a fraction $w_{\mathrm{F}}(t)$ of its total assets in the risky asset, where $w_{\mathrm{F}}(t)$ follows a random process that reverts to the fundamental value $\mu$. (2) We allow random innovations in the portfolio weight that reflect exogenous shocks. This is done by making the noise term a GARCH $(1,1)$ process. Thus, the fundamentalist investor provides a source of time varying exogenous volatility to the model, to which the bank reacts by adjusting its estimate of risk and consequently its leverage.

Given the aggregate demand of the bank and the fund, and assuming for simplicity that there is a supply of exactly one unit of the risky asset that is infinitely divisible, the price of the risky asset is determined through market clearing by equating demand and supply.

\subsection{Time evolution}

The model evolves in discrete time-steps of length $\tau$. We make this a free parameter so that the model has well-defined dynamics in the continuum limit $\tau \rightarrow 0$, which is useful for calibration. At each time-step the bank and the fund update their balance sheets as follows:

- The bank updates its historically-based estimate of future volatility and computes its new target leverage accordingly. Volatility estimation is done using an exponential moving average with an approach similar to RiskMetrics (see Longerstaey (1996)).

- The bank pays dividends or raises capital to reach its target equity $\bar{E}$. 
- The bank determines how many shares of the risky asset it needs to trade to reach its target leverage.

- At the same time, the fundamentalist fund submits its demand for the risky asset.

- The market clearing price for the risky asset is computed and trades occur. ${ }^{14}$

\subsection{The model as a dynamical system}

The dynamics of our model can be described as an iterated map for the state variable $x(t)$, defined as

$x(t)=\left[\sigma^{2}(t), w_{\mathrm{F}}(t), p(t), n(t), L_{\mathrm{B}}(t), p^{\prime}(t)\right]^{\mathrm{T}}$.

$\sigma$ is the historical estimation of the volatility of the risky asset; $w_{\mathrm{F}}$ is the fraction of wealth invested by the fund in the risky asset; $p$ is the current price of the risky asset; $n$ is the share of the risky asset owned by the bank; $L_{\mathrm{B}}$ are the liabilities of the bank; and $p^{\prime}$ is the lagged price of the asset, i.e. the price at the previous time-step. A detailed derivation of the model is presented in Appendix A. Here we simply present the model and provide some basic intuition. Let us introduce the following definitions:

Bank assets :

$A_{\mathrm{B}}(t)=p(t) n(t) / w_{\mathrm{B}}$,

Target leverage :

$$
\bar{\lambda}(t)=\alpha\left(\sigma^{2}(t)+\sigma_{0}^{2}\right)^{b},
$$

Leverage adjustment : $\Delta B(t)=\tau \theta\left(\bar{\lambda}(t)\left(A_{\mathrm{B}}(t)-L_{\mathrm{B}}(t)\right)-A_{\mathrm{B}}(t)\right)$,

Equity redistribution : $\kappa_{\mathrm{B}}(t)=-\kappa_{\mathrm{F}}(t)=\tau \eta\left(\bar{E}-\left(A_{\mathrm{B}}(t)-L_{\mathrm{B}}(t)\right)\right)$,

Bank cash :

$c_{\mathrm{B}}(t)=\left(1-w_{\mathrm{B}}\right) n(t) p(t) / w_{\mathrm{B}}+\kappa_{\mathrm{B}}(t)$,

Fund cash :

$$
c_{\mathrm{F}}(t)=\left(1-w_{\mathrm{F}}(t)\right)(1-n(t)) p(t) / w_{\mathrm{F}}(t)+\kappa_{\mathrm{F}}(t) .
$$

$A_{\mathrm{B}}$ is the assets of the bank, $\kappa_{\mathrm{B}}$ is the equity adjustment of the bank on a given time-step, $\kappa_{\mathrm{F}}$ is the equity adjustment of the fund, $w_{\mathrm{B}}$ is the portfolio weight of the bank, $\bar{E}$ is the bank's equity target, $c_{\mathrm{B}}$ is the bank's cash and $c_{\mathrm{F}}$ is the fund's cash. The parameters $\theta$ and $\eta$ determine how aggressive the bank is in reaching its targets for leverage and equity, i.e. the bank aims at reaching the targets on time horizons of the order $1 / \theta$ and $1 / \eta$.

The model can be written as a dynamical system in the form

$x(t+\tau)=g(x(t))$

where the function $g$ is the following 6-dimensional map:

$$
\begin{aligned}
& \sigma^{2}(t+\tau)=(1-\tau \delta) \sigma^{2}(t)+\tau \delta\left(\log \left[\frac{p(t)}{p^{\prime}(t)}\right] \frac{t_{\mathrm{VaR}}}{\tau}\right)^{2}, \\
& W_{\mathrm{F}}(t+\tau)=W_{\mathrm{F}}(t)+\frac{W_{\mathrm{F}}(t)}{p(t)}[\tau \rho(\mu-p(t))+\sqrt{\tau} s(t) \xi(t)], \\
& p(t+\tau)=\frac{w_{\mathrm{B}}\left(c_{\mathrm{B}}(t)+\Delta B(t)\right)+w_{\mathrm{F}}(t+\tau) c_{\mathrm{F}}(t)}{1-w_{\mathrm{B}} n(t)-(1-n(t)) w_{\mathrm{F}}(t+\tau)}, \\
& n(t+\tau)=\frac{w_{\mathrm{B}}\left(n(t) p(t+\tau)+c_{\mathrm{B}}(t)+\Delta B(t)\right)}{p(t+\tau)}, \\
& L_{\mathrm{B}}(t+\tau)=L_{\mathrm{B}}(t)+\Delta B(t), \\
& p^{\prime}(t)=p(t+\tau) .
\end{aligned}
$$

\footnotetext{
${ }^{14}$ It is important to note that the decision concerning equity and investment adjustments is taken before the current trading price of the risky asset is revealed. We therefore assume that the bank uses the price of the previous time-step as a proxy for the expected trading price, and acts accordingly. This assumption of myopic expectations marks a significant departure of our model from the general equilibrium setting of Adrian and Boyarchenko (2012) and Adrian and Boyarchenko (2013), but it is common in the literature on heterogeneous agents in economics (see for instance Hommes (2006)).
}

Each of these equations can be understood as follows:

(a) The expected volatility $\sigma^{2}$ of the risky asset is updated through an exponential moving average. The parameter $\tau \delta \in(0,1)$ defines the length of the time-window over which the historical estimation is performed, while the parameter $t_{\mathrm{VaR}}$ represents the time-horizon used by the bank in the calculation of VaR.

(b) The adjustment of the fund's risky asset portfolio weight $w_{\mathrm{F}}$ drives the price towards the fundamental value $\mu$, with an adjustment rate $\tau \rho \in(0,1)$. The demand of the fund also depends on exogenous noise, which is assumed to be a normal random variable $\xi(t)$ with amplitude $s(t) \geq 0$. The amplitude varies in time so that the variable $\chi(t)=s(t) \xi(t)$ follows a GARCH $(1,1)$ process. The factors of $\tau$ guarantee the correct scaling as $\tau \rightarrow 0$.

(c) The market clears.

(d) The bank ownership of the risky asset $n(t+1)$ adjusts according to market clearing.

(e) Bank liabilities are updated to account for the change $\Delta B(t)$ in the asset side of the balance sheet.

(f) Due to the dependence of Eq. (5d) on the lagged price $p(t+\tau)$, we must define an additional variable $p^{\prime}(t)$ to make the map a first order dynamical system.

\section{Overview of model behavior}

In order to explore the dynamical behavior of the model, we solve it numerically. We begin by studying fully procyclical leverage control policies corresponding to risk management under VaR, i.e. we choose $b=-0.5$ throughout this section. A summary of the parameters is provided in Table 1.

\subsection{How we chose parameters}

While this model is too stylized to expect a perfect match to real data, it has the advantage that its most important parameters can be estimated a priori, and once these parameters are fixed its behavior is fairly robust for reasonable values of the other parameters. In the following we briefly discuss how we choose the key parameters and how they affect the behavior.

\section{Timescale parameters}

The first five parameters listed in Table 1 play a dominant role in determining the timescale of the cycle that we observe. We have carefully constructed the model so that it reaches a continuum limit

Table 1

Overview of parameters for the numerical model solution.

In the default column, a "(v)" indicates that this is the default value, but that the parameter is sometimes varied (as noted in the text); a unit of " 1 " indicates that the parameter is dimensionless and $\$$ that it has monetary units.

\begin{tabular}{lllll}
\hline & Symbol & Description & Default & Unit \\
\hline Bank & $\tau$ & Time-step & 0.1 & year \\
& $\delta$ & Memory for volatility estimation & 0.5 & year $^{-1}$ \\
& $t_{\text {VaR }}$ & Horizon for VaR calculation & 0.1 & year \\
& $\theta$ & Leverage adjustment speed & $10(\mathrm{v})$ & year $^{-1}$ \\
& $\eta$ & Equity redistribution speed & 10 & year $^{-1}$ \\
& $b$ & Cyclicality of leverage control & $-0.5(\mathrm{v})$ & 1 \\
& $\sigma_{0}^{2}$ & Risk offset & $10^{-6}$ & 1 \\
& $\alpha$ & Risk level & $0.075(\mathrm{v})$ & 1 \\
& $\bar{E}$ & Bank's equity target & $2.27(\mathrm{v})$ & $\$$ \\
& $w_{\mathrm{B}}$ & Bank's weight for risky asset & $0.3(\mathrm{v})$ & 1 \\
Fund & $\mu$ & Fundamental value & 25 & $\$$ \\
& $\rho$ & Mean reversion & 0.1 & year $^{-1}$ \\
GARCH & $a_{0}$ & Baseline return variance & $10^{-3}$ & 1 \\
& $a_{1}$ & Error autoregressive term & 0.016 & 1 \\
& $b_{1}$ & Variance autoregressive term & 0.87 & 1 \\
\hline
\end{tabular}


as the elementary time-step $\tau \rightarrow 0$. For computational efficiency we choose $\tau$ to be the largest possible value with behavior similar to that in the continuum limit, which results in a time-step of $\tau=0.1$ years. As long as $\tau$ is this size or smaller the results change very little.

The parameter $\delta$ sets the timescale for the exponential moving average used to estimate volatility, and is the most important determinant of the overall timescale of the dynamics. The characteristic time for the moving average is $t_{\delta}=1 / \delta .{ }^{15}$ According to the RiskMetrics approach Longerstaey (1996), the typical timescale used by market practitioners is $t_{\delta} \approx 2$ years. We therefore set $\delta=0.5$ year $^{-1}$, corresponding to a two year timescale, and keep it fixed throughout.

The parameter $t_{\mathrm{VaR}}$ is the time horizon over which returns are computed for regulatory purposes. In practice this varies depending on the liquidity of the asset portfolio and ranges from days to years. A good rule of thumb is to choose $t_{\mathrm{VaR}}$ roughly equal to the time needed to unwind the portfolio. We assume $t_{\mathrm{VaR}}=\tau=0.1$ years, i.e. a little more than a month. Changing the level of $t_{\mathrm{VaR}}$ essentially scales the level of perceived risk. Therefore, via the bank's leverage, the effects of $t_{\mathrm{VaR}}$ and $\alpha$ on the model dynamics are tightly linked. An increase in $t_{\mathrm{VaR}}$ is equivalent to a corresponding decrease in $\alpha$.

The parameters $\theta$ and $\eta$ define how aggressive the bank is in reaching its targets for leverage and equity. Our default assumption is that the bank tries to meet its target on a timescale of about one time-step of the dynamics, and so unless otherwise stated, in the following we set $\theta=10$ year $^{-1}$ and $\eta=10$ year $^{-1}$. This ensures that the bank's realized leverage is always close to its target. We will vary the parameter $\theta$ and discuss how it affects the stability of the dynamics in Section 4.3.

The mean reversion parameter $\rho$ determines the aggressiveness with which the fund responds to deviations in the price of the risky asset from its fundamental value $\mu$. We take $\rho=0.1$, i.e. an adjustment rate for the fund's weight $w_{\mathrm{F}}$ of the risky asset of about $10 \%$ per time-step $\tau$; see Equation (5b). This parameter does not affect the dynamics very much as long as $\rho<1$. In the extreme case when $\rho$ becomes large and the fund has sufficient market power, price deviations from the fundamental value will become small. Conversely, when $\rho$ is very small, price deviations from the fundamental value will be allowed to become large and the system will become more unstable.

\section{Market impact of the bank}

The dynamics of this model depend on the competition between the stabilizing properties of the fundamentalist and the destabilizing properties of the bank. The market impact of the bank is roughly speaking the product of the leverage $\lambda$ and the relative size of the banking sector $R$.

The target leverage is proportional to $\alpha$, with $\sigma_{0}$ determining an upper bound when $b<0$, as given in Eq. (2). We take $\sigma_{0}=10^{-3}$, which means that the upper bound is seldom met, and control the leverage by varying $\alpha$. A typical value of $\alpha$ that yields a leverage cycle is $\alpha=0.075$.

The relative size of the bank $R$ is important in determining the stability of the model. We show in Eq. (A.10) in Appendix A that at the fixed point equilibrium the parameters $\bar{E}, w_{\mathrm{B}}, \mu, \sigma_{0}$, and $\alpha$, as well as the initial condition $w_{\mathrm{F}}(0)$, jointly determine the fraction of the risky asset $R$ owned by the bank. The numerical values chosen for the target equity $\bar{E}$ and the fundamental price $\mu$ are arbitrary only the ratio between them is important. We choose the ratio $\bar{E} / \mu$, the bank's portfolio weight $w_{\mathrm{B}}$ and the fund's initial weight $w_{\mathrm{F}}(0)$

\footnotetext{
15 The contribution to the moving average of a squared return $y(t)$ observed at time $t$ is $y(t+\Delta t)=(1-\tau \delta)^{\Delta t / \tau} y(t)$ at time $t+\Delta t$. We define the typical time $t_{\delta}$ such that $y\left(t+t_{\delta}\right) / y(t)=1 / e$. Thus, $t_{\delta}=-\tau / \log [1-\tau \delta] \approx 1 / \delta$ for $\tau \delta \ll 1$.
}
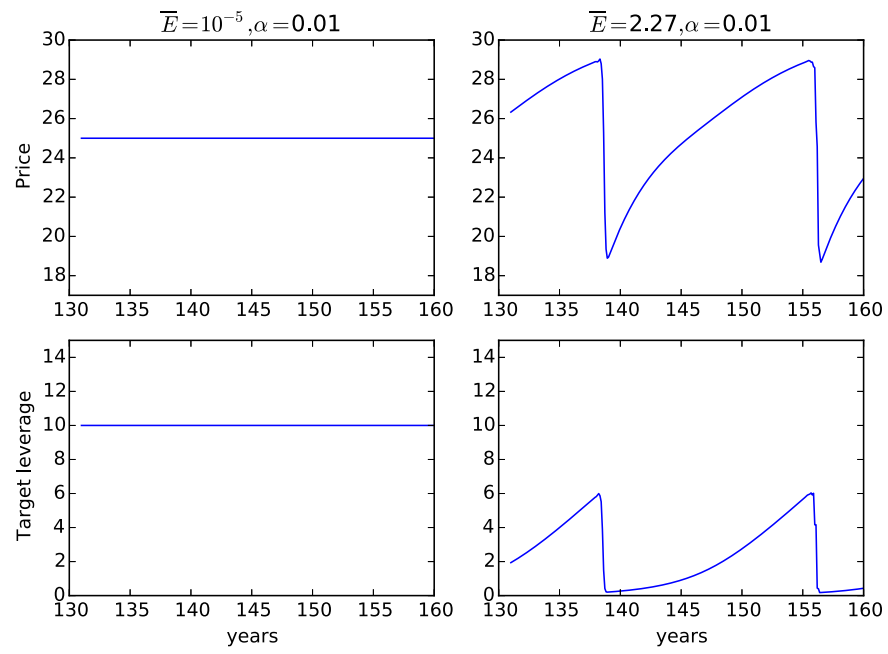

Fig. 4. Time series of price and leverage in the deterministic case. Left panel: scenario (i) - microprudential, the fund dominates the bank $\left(\bar{E}=10^{-5}\right)$, i.e. the bank has no significant market impact. In this case, the system goes to a fixed point equilibrium where the leverage and price of the risky asset remain constant. Right panel: scenario (ii) - macroprudential, the bank has significant market impact $(\bar{E}=2.27)$. In this case, the bank's risk management leads to persistent oscillations in leverage and price of the risky asset with a time period of roughly 15 years.

in order to get a sensible value for the relative size of the bank, and use $\bar{E}$ to vary this as needed. Bear in mind that the bank represents all investors with leverage targets.

Finally, we pick parameters for the fund GARCH process $a_{0}, a_{1}$ and $b_{1}$ in order to achieve a reasonable level of clustered volatility and to permit leverage cycles even in the presence of noise as observed in Fig. 1.

\subsection{Overview of model dynamics}

We now build some intuition about the model dynamics. First, consider the extreme case where $\bar{E} \rightarrow 0$, i.e. where the market impact of the bank is negligible so that the price dynamics are dominated by the fund. This is the purely microprudential case where the bank's actions have no significant effect on the market. With $s>0$ the exogenous volatility perturbs the system away from its equilibrium and the price performs a mean reverting random walk around the fundamental price $\mu$. In the deterministic case, i.e. $s=0$, the fund quickly drives the dynamics to the fundamental price and the system settles to a fixed point equilibrium.

When the bank's equity target $\bar{E}$ is large enough that the bank has a significant impact on the price process, the dynamics are less straightforward. We refer to this scenario as the macroprudential case. The destabilizing market impact of the bank can drive chaotic endogenous oscillations, as described below, which introduce endogenous volatility on top of exogenous volatility.

We investigate the following four scenarios:

(i) Deterministic, microprudential: $\bar{E}=10^{-5}$ and $s=0$.

(ii) Deterministic, macroprudential: $\bar{E}=2.27$ and $s=0$.

(iii) Stochastic, microprudential: $\bar{E}=10^{-5}$ and $s>0$.

(iv) Stochastic, macroprudential: $\bar{E}=2.27$ and $s>0$.

Unless otherwise stated, all parameters are as specified in Table 1. The first two cases are for the deterministic limit with $s=0$. Although the deterministic limit is unrealistic, it is useful to gain intuition, and in particular to understand the nature and origin of the endogenous oscillation observed in cases (ii) and (iv). The last two cases are with more realistic levels of exogenous noise. We 

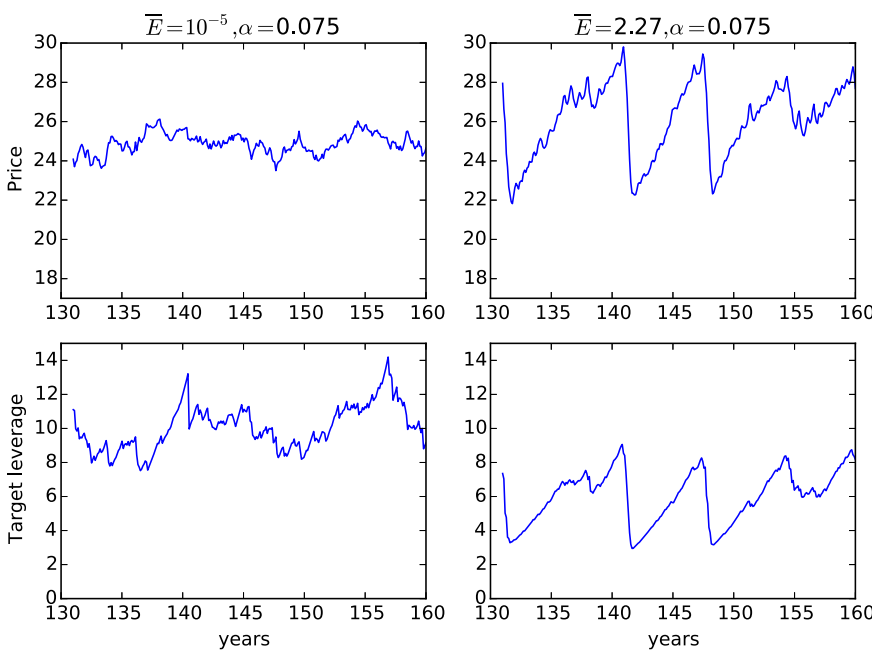

Fig. 5. Time series of price and leverage in the stochastic case. Left panel: scenario (iii) - microprudential, the fund dominates the bank $\left(\bar{E}=10^{-5}\right)$, i.e. the bank has no significant market impact. In this case, the price is driven by the fund's trading activity and performs a mean reverting random walk around the fundamental value $\mu=25$. Right panel: scenario (iv) - macroprudential, the bank has significant market impact $(\bar{E}=2.27)$. In this case, the bank's risk management leads to irregular oscillations in leverage and price of the risky asset that are similar to the deterministic case.

summarize our results for scenarios (i) and (ii) in Fig. 4 and for scenarios (iii) and (iv) in Fig. 5.

The microprudential scenarios (i) and (iii) behave as expected: In the deterministic limit the system simply settles into a fixed point with prices equal to fundamental values. When there is exogenous noise the system makes excursions away from the fixed point but never drifts far away from it, and the dynamics remain relatively simple.

In contrast, the macroprudential scenarios (ii) and (iv) display large oscillations both in leverage and price. We refer to this oscillation as the Basel leverage cycle. Notably, the oscillations occur even in the deterministic limit, i.e. without any external shocks. During the cycle the price and leverage slowly rise and then suddenly fall, with a period of about $\Delta t \approx 15$ years in the deterministic case.

The oscillations have the following economic interpretation, which is easiest to understand in the deterministic limit: Suppose we begin at about $t=140$ years in the right panels of Fig. 4 , with leverage low, perceived risk high, and prices low but increasing. ${ }^{16}$ Going forward in time the perceived risk slowly decreases as the memory of the past crisis fades. From a mechanical point of view, this is due to the smoothing action of the exponential moving average - as long as the change in price is lower than the current historical average the volatility $\sigma^{2}$ continues to drop. This causes the leverage to increase under the procyclical leverage policy. The bank buys more shares to meet its new leverage target, driving prices up. This process continues for many years, generating a gradual rise in both prices and leverage. The system slowly approaches its equilibrium, but the equilibrium is now unstable due to the high leverage. Eventually, the amplification of price due to leverage is sufficiently large that the volatility begins to increase. This drives the leverage down, which causes selling, driving prices down and volatility up. The strong positive feedback generates a sharp crash that ultimately comes to an end due to the increasingly heavy investment of the fundamentalist fund. After the crash volatility is high and leverage is low, and the cycle repeats itself, except that

\footnotetext{
16 We begin at a time where $t$ is substantially greater than zero in order to let transients die out so that the system has settled onto its attractor.
}

because the dynamics are chaotic the precise details of the subsequent "Great Financial Moderations" and crashes vary from cycle to cycle.

From a dynamical systems point of view, the equilibrium is a hyperbolic fixed point. ${ }^{17}$ During the "Great Financial Moderation", when prices are steadily rising, the system approaches the equilibrium along its stable manifold. However, because it never approaches it exactly, it eventually veers away and exits along the unstable manifold, generating a crisis.

The behavior in the stochastic case is similar, except that, in addition to generating an endogenous oscillation, the instabilities creating the chaotic behavior also strongly amplify the exogenous noise. This creates the possibility that chance events may significantly modify the basic cycle.

We stress that this behavior is not due to a fine tuning of the model specifications, but rather appears to be a robust property that emerges from the combination of a historical estimation of risk, and an active portfolio management that is based on VaR. We have observed similar cycles in different models, ranging from an extremely simple two dimensional map as given in Aymanns and Farmer (2015), to more complex settings involving multiple banks and assets, cf. Aymanns and Farmer (2015).

The fragility that drives the crashes comes from the fact that at high levels of leverage a small increase in risk is sufficient to cause a drastic tightening of the leverage constraint. This intuition can be made precise by comparing the derivative of the leverage control policy for high vs. low leverage. For convenience, we take $\sigma_{0}^{2} \ll 1$, which under the assumption that $b=-1 / 2$ means that leverage is very large. ${ }^{18}$ The result is that

$$
\begin{aligned}
& \frac{d F_{\left(\alpha, \sigma_{0}^{2},-0.5\right)}}{d \sigma^{2}(t)}\left(\sigma^{2}(t)\right) \\
& = \begin{cases}-0.5 / \sigma_{0}^{3} \ll 0 & \text { for } \sigma^{2}(t) \rightarrow 0 \wedge \sigma_{0}^{2} \ll 1, \\
0 & \text { for } \sigma^{2}(t) \rightarrow \infty \wedge \sigma_{0}^{2} \ll 1 .\end{cases}
\end{aligned}
$$

In the high leverage limit, i.e. when $\sigma$ is small, the sensitivity of the leverage target $F$ to variations in risk tends to infinity. In contrast, the sensitivity is zero in the opposite limit where leverage is low and perceived risk is large. Thus, when the leverage is high, only a small increase in volatility is needed to cause a large change in leverage, causing a large effect on prices, which further increases volatility, creating a feedback loop that suddenly drives leverage and prices down. Therefore, increasing leverage of the banking system has a two-fold destabilizing effect: It makes the dynamics unstable and leads to chaos, but it also makes the system more sensitive to shocks, which can result in sudden deleveraging triggered by external events (such as the collapse of a housing bubble).

The leverage cycles are not strictly periodic due to the fact that the oscillations are chaotic. This becomes clearer by plotting the dynamics in phase space and then taking a Poincaré section, as illustrated in Fig. 6. The phase plot makes the cyclical structure clearer. The 3D representation in the left panel shows how the ownership of the risky asset $n$, the perceived risk $\sigma$ and the price $p$ vary during the course of the leverage cycle. The Poincaré section in the right panel is constructed by plotting ownership vs. perceived risk every time the trajectory crosses the hyper-plane $p(t)=20$ with the price increasing. The Poincaré section shows the characteristic fractal structure, and shows the stretching and folding that makes the

\footnotetext{
17 A hyperbolic fixed point is a fixed point that is unstable in some directions but stable in others. Loosely speaking the stable manifold is the set of points that asymptotically reach the fixed point and the unstable manifold is the set corresponding to iteration of the unstable eigenvector.

18 For $b<0$ the parameter $\sigma_{0}$ imposes a cap on the target leverage; larger values for $\sigma_{0}^{2}$ would make this unrealistically low.
} 

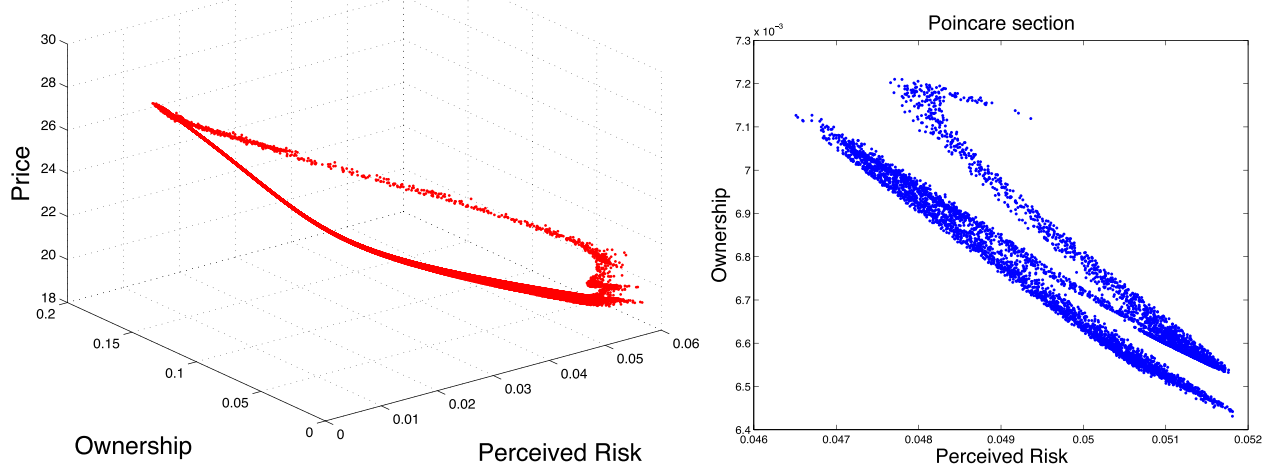

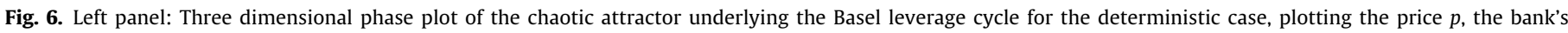

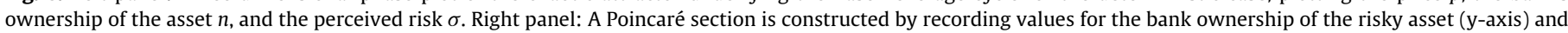

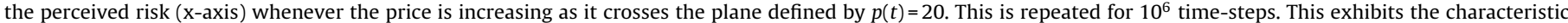
stretching and folding associated with chaotic dynamics.

dynamics chaotic. The fact that these dynamics are chaotic is confirmed in the next section, where we do a stability analysis and compute the Lyapunov exponent.

In summary, depending on the choice of parameters, the model either goes to a fixed point (scenario (ii)) or shows chaotic irregular cycles (scenarios (i) and (iii)). As expected, the dynamics become more complicated when noise is added, but the essence of the Basel leverage cycle persists even in the zero noise limit.

\section{Determinants of model stability}

\subsection{Deterministic case}

In the deterministic case the standard tools of linear stability analysis can be used to characterize the boundary between the fixed point equilibrium and leverage cycles. In this section we will use this to characterize the behavior of the system as the risk parameter $\alpha$ and the cyclicality parameter $b$ are varied. We begin by studying the deterministic case, where we can draw analytical insights, and then present numerical results for the stochastic case. The details of the stability analysis are presented in Appendix A.

The dynamical system has a unique fixed point equilibrium $x^{*}$, given by

$$
\begin{aligned}
x^{*} & =\left(\sigma^{2 *}, w_{\mathrm{F}}^{*}, p^{*}, n^{*}, L_{\mathrm{B}}^{*}, p^{\prime *}\right) \\
& =\left(0, w_{\mathrm{F}}(0), \mu, \frac{1}{\mu} \alpha \sigma_{0}^{2 b} \bar{E} w_{\mathrm{B}},\left(\alpha \sigma_{0}^{2 b}-1\right) \bar{E}, \mu\right) .
\end{aligned}
$$

This corresponds to a leverage $\lambda^{*}$ and relative size of bank to fund $R\left(x^{*}\right)$, given by

$$
\begin{aligned}
\lambda^{*} & =\alpha \sigma_{0}^{2 b}, \\
R\left(x^{*}\right) & =\frac{A_{\mathrm{B}}^{*}}{A_{\mathrm{F}}^{*}}=\frac{\lambda^{*} E_{\mathrm{B}}^{*}}{\left(1-n^{*}\right) p^{*} / w_{\mathrm{F}}^{*}} .
\end{aligned}
$$

At the equilibrium $x^{*}$ the price is constant at its fundamental value and the bank is at its target leverage. The stability of the equilibrium depends on the parameters. Regime (i) observed in the numerical simulations of the previous section corresponds to the stable case. In this case, regardless of initial conditions, the system will asymptotically settle into the fixed point $x^{*}$. In contrast, when the fixed point $x^{*}$ is unstable there are two possibilities. One is that there is a leverage cycle, in which the dynamics are locally unstable but exist on a chaotic attractor that is globally stable; the other is that the system is globally unstable, in which case the price either becomes infinite or goes to zero.

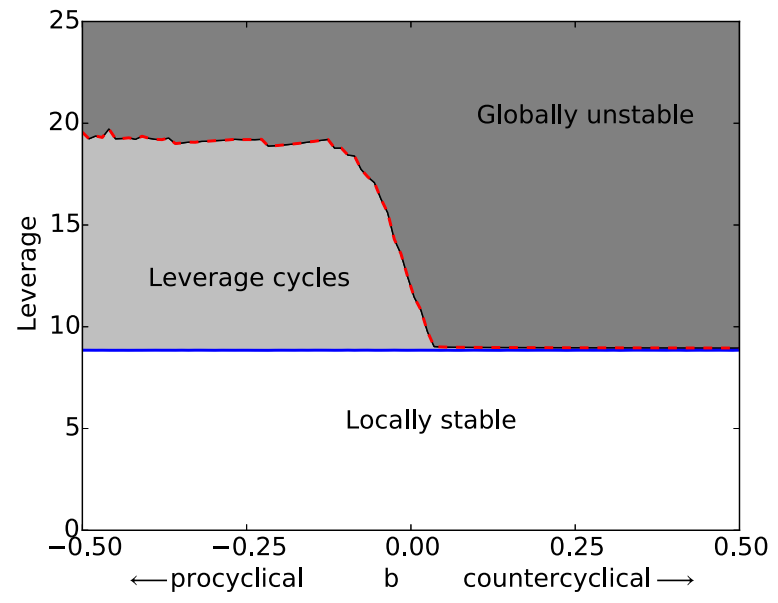

Fig. 7. A bifurcation diagram showing the three regimes in the deterministic case. The risk parameter $\alpha$ and the cyclicality parameter $b$ are varied while holding the other parameters constant at the value in Table 1 . The white region corresponds to a stable fixed point equilibrium, the light gray region to leverage cycles and the dark gray region to global instability. The solid blue line corresponds to the critical leverage $\lambda_{c}^{*}$ in Eq. (7) at the critical value $\alpha_{c}$ where the fixed point becomes unstable. (For interpretation of the references to color in this figure legend, the reader is referred to the web version of this article.)

In Fig. 7, we show the results of varying the risk parameter $\alpha$ and the cyclicality parameter $b$. The risk parameter $\alpha$ provides the natural way to vary the risk of the bank, but the realized risk for a given $\alpha$ depends on other factors such as changes in volatility. For diagnostic purposes, leverage is a better measure. ${ }^{19}$ Fig. 7 shows each of the three regimes, corresponding to (i) stable equilibrium, (ii) leverage cycles and (iii) global instability, as a function of the leverage and the cyclicality parameter $b .^{20}$

This diagram reveals several interesting results. As expected, for low leverage the system is stable and for higher leverage it is unstable. Somewhat surprisingly, the critical leverage $\lambda_{c}^{*}$ is independent of $b$, and consequently the size of the regime with the

\footnotetext{
19 While $\alpha$ tends to increase leverage, when the leverage control policy is procyclical the behavior is not always monotonic. This is because increasing $\alpha$ tends to increase volatility, but increasing volatility drives the target leverage down, so the two effects compete with each other.

20 The boundary where the fixed point equilibrium becomes unstable is computed analytically based on the leverage $\lambda_{c}^{*}$ where the modulus of the leading eigenvalue is one. The boundary for globally unstable behavior is more difficult to compute as it requires numerical simulation.
} 
stable equilibrium is unaffected by whether the leverage control is procyclical or countercyclical. In the procyclical regime $(b<0)$, there is a substantial region with leverage cycles. Note that this transition occurs suddenly, i.e. if $\alpha$ is increased by a small amount which happens to cross the threshold, the system suddenly moves from a fixed point to a large, finite amplitude oscillation. Thus, a small regulatory change can result in a dramatic difference in outcomes.

For the countercyclical regime $(b>0)$, the system makes a direct transition from the stable fixed point equilibrium to global instability throughout most of the parameter range. The instability is not surprising: In the countercyclical regime, there is an unstable feedback loop in which increasing leverage drives increasing prices and increasing volatility, which further increases the leverage. Thus, for high leverage there are unstable regimes for both pro- and countercyclical behavior, but when it occurs, the instability is more severe in the countercyclical regime.

\subsection{Stability when there is exogenous noise}

When there is exogenous noise we can only measure the stability numerically. This is done by computing the largest Lyapunov exponent of the dynamics. The Lyapunov exponents are a generalization of eigenvalues for limit cycles and chaotic attractors. The leading Lyapunov exponent measures the average rate at which the separation between two nearby points changes in time. When the dynamics are locally stable, nearby points converge exponentially and the leading Lyapunov exponent is negative, and when they are locally unstable, nearby points diverge exponentially and the leading Lyapunov exponent is positive. The Lyapunov exponent is a property of a trajectory, but for dissipative systems such as ours, almost all trajectories on a given attractor have the same Lyapunov exponents, so it is a property of the attractor. If the attractor is a fixed point, the largest Lyapunov exponent is negative, and if it is a chaotic attractor, the largest Lyapunov exponent is positive. As expected, in the deterministic case we observe that leverage cycles have a positive leading Lyapunov exponent, confirming that the dynamics are chaotic.

It is also possible to compute Lyapunov exponents for stochastic dynamics. To understand the basic idea of how this is done, imagine two realizations of the dynamics with the same sequence of random shocks, but starting at slightly different initial conditions (see Crutchfield et al. (1982)). Because the random noise is the same in both cases, it is possible to follow two infinitesimally separated points and measure the rate at which they separate. If the leading Lyapunov exponent is positive this means that the dynamics will strongly amplify the noise.

We compare the stability for the stochastic and deterministic cases in Fig. 8. This is done for the procyclical case only, since the direct transition from a fixed point to global instability in the countercyclical case complicates numerical work (and the countercyclical case is less relevant for our analysis).

In the stochastic case, the critical leverage is computed as the time average of the target leverage when the Lyapunov exponent becomes positive. Interestingly, the critical leverage in the stochastic case first starts below the deterministic critical leverage and then approaches it as $b$ is increased. This indicates that for strongly procyclical leverage control policies noise destabilizes the system. The gray line in Fig. 8 shows the average target leverage used for the evaluation of the optimal leverage control policy in Section 5. In this exercise the average target leverage was intentionally held fix across different values of $b$.

The most interesting conclusion from comparing the stochastic and deterministic cases is that when the dynamics are strongly procyclical (i.e. for $-0.5<b<-0.2$ ) the noise significantly lowers the stability threshold. In contrast, for larger values of $b>-0.2$ there is little difference in the stability threshold in the two cases. This

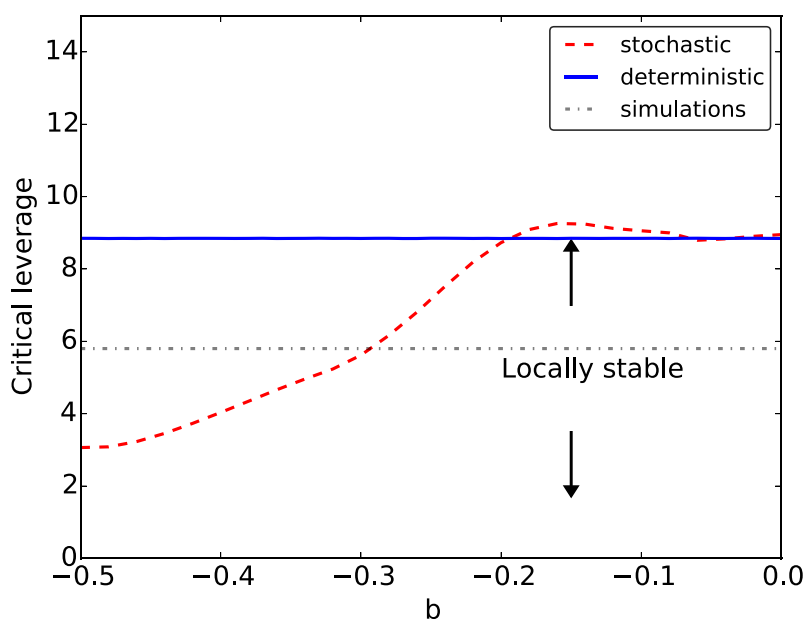

Fig. 8. A comparison of stability when the dynamics are deterministic vs. stochastic for the procyclical region $(b<0)$. As in the previous figure, the critical leverage $\lambda_{c}^{*}$ for the deterministic case is shown as a blue line. The dashed red line shows the parameter value where the dynamics become unstable as measured by the leading Lyapunov exponent; note the transition to chaos occurs at a much lower leverage. The gray line show the average target leverage used for the evaluation of the optimal leverage control policy in Section 5. (For interpretation of the references to color in this figure legend, the reader is referred to the web version of this article.)

indicates that the dynamics becomes more stable when the leverage control policy is close to constant leverage. This, together with the fact that in the countercyclical regime the system goes straight from stability to global instability, suggests that intermediate values of cyclicality (nearer to constant leverage) are likely to be most stable, at least when all the parameters as chosen as in Table 1.

\subsection{Slower adjustment leads to greater stability}

The bank's leverage adjustment speed $\theta$ has a strong effect on stability, with interesting regulatory implications. Intuitively, in the macroprudential regime, decreasing the adjustment speed means less aggressive selling during deleveraging, which should make the system more stable. ${ }^{21}$

To test this we study how the critical leverage $\lambda_{c}^{*}$ and critical relative size $R_{c}\left(x^{*}\right)$ depend on the adjustment speed $\theta \tau$ (we vary $\theta$ and hold $\tau$ constant). The relationship is shown in Fig. 9, where the critical leverage is shown on the left vertical axis and the critical relative size on the right vertical axis. As expected, both the critical leverage (left axis, continuous line) and critical relative size of the bank (right axis, dashed line), decrease dramatically as $\theta \tau$ increases. This suggests that it is possible to dramatically improve the stability of the financial system if financial institutions adjust to their leverage targets slowly. Similarly, this illustrates the dangers of mark-to-market accounting, which can cause balance-sheet adjustments to be too rapid.

\subsection{Longer risk estimation horizon increases stability}

Another important policy parameter is the horizon over which banks estimate their perceived risk. The estimation horizon is given by the characteristic time of the exponential moving average for

\footnotetext{
21 We have considered the case where the bank increases its leverage quicker than it decreases it. We have done this introducing an asymmetry in the parameter $\theta$ that controls the speed of leverage adjustment, i.e. introducing a parameter $\theta_{+}$for the speed of levering up and a parameter $\theta_{-}$for the speed of deleveraging. By allowing such asymmetric specification, we find that the dynamics becomes more stable as $\theta_{-}$is reduced. The qualitative behavior of the system, namely the existence of stable, locally unstable and globally unstable regimes, is preserved.
} 


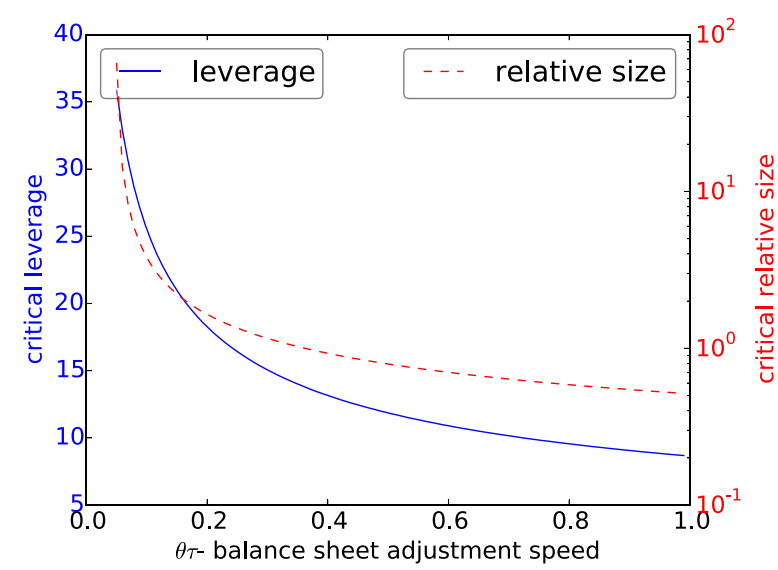

Fig. 9. Critical leverage $\lambda_{c}^{*}$ (solid blue line, left vertical axis) and the critical value of the relative size of the bank to the fund $R_{c}\left(x^{*}\right)$ (dashed red line, right vertical axis) as a function of the leverage adjustment speed $\theta \tau$. Other parameters are as in Table 1. The stability of the financial system can be dramatically improved by lowering the adjustment speed. (For interpretation of the references to color in this figure legend, the reader is referred to the web version of this article.)

the risk estimation $t_{\delta}=1 / \delta$. The larger $t_{\delta}$ the slower the bank will update its perceived risk as it observes new price movements. At the same time the memory of past crisis will persist longer. Therefore, one could argue that a bank with a large risk estimation horizon $t_{\delta}$ follows a "long-term" risk management strategy while a bank with a small $t_{\delta}$ follows a "short-term" risk management policy.

In order to test how the risk estimation horizon affects the stability of the system we compute the critical leverage $\lambda_{c}^{*}$ for different values of $t_{\delta}$ in the stochastic case (i.e. via determining when the leading stochastic Lyapunov exponent becomes positive). We find that the critical leverage increases monotonically in the risk estimation horizon $t_{\delta}$. For small risk estimation horizons ( $t_{\delta} \approx 1$ year), the critical leverage increases very quickly with a small change $t_{\delta}$. As $t_{\delta}$ increases the rate of increase of the critical leverage slows down such that for $t_{\delta}>7$ years the rate of increase in the critical leverage becomes insignificant. This suggests that within our model, given sufficiently short initial risk estimation horizons, a policy maker may significantly improve the financial system's stability by mandating long term risk management policies.

\section{Leverage control policies}

What is the optimal leverage control policy? The mere fact that the endogenous oscillations of prices and volatility depend on the cyclicality parameter $b$, as shown in Fig. 7, suggests that some policies are better than others. In this section we introduce a procedure for scoring policies and search for the best policy within the family that we have defined. We find that the optimal policy depends on parameters of the model, and in particular on the market impact of banks in relation to the rest of the financial system. For the parameters used in Table 1, as the market impact of banks increases the optimal policy becomes increasingly less procyclical, and in the limit where the banks play a large role in determining prices it approaches constant leverage.

\subsection{Criterion for optimality}

We define an optimal leverage control policy as one that maximizes leverage for a given level of risk. Maximizing leverage is desirable because it means that, for a given level of capital, banks are able to lend more money. We do not model the real economy here, we simply take it as a given that the ability to obtain credit if needed

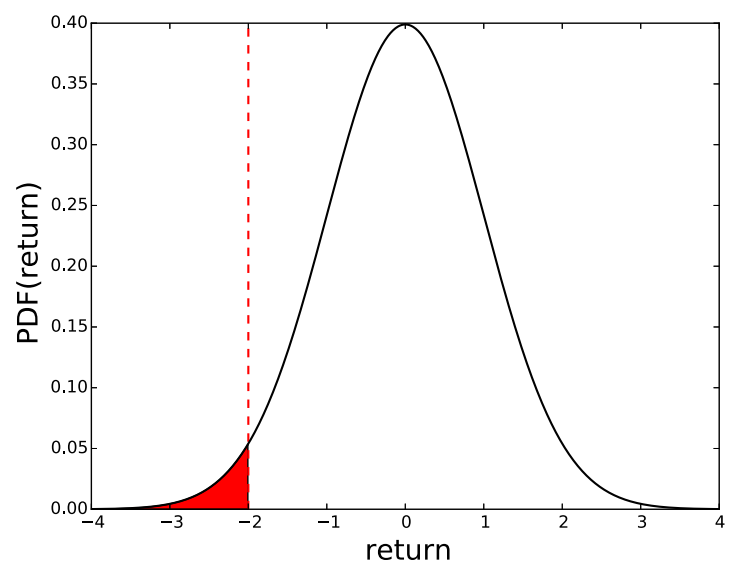

Fig. 10. Visual representation of realized shortfall. The solid black lines represents a hypothetical return distribution. The red vertical dashed line is drawn in correspondence of the q-quantile of the distribution. The realized shortfall is the average of the distribution in the red region to the left of the vertical line. (For interpretation of the references to color in this figure legend, the reader is referred to the web version of this article.)

is desirable for the real economy. ${ }^{22}$ From a practical point of view, it is difficult to control risk while searching the parameter space. It is much easier to control the average leverage, systematically sweep parameters and measure the resulting risk.

To measure risk we have the luxury of having a simple model, which we can iterate numerically to generate as much data as we need for statistical estimation. We can then observe the resulting time series of gains and losses for the bank and measure the level of risk associated with this time series. Because this is an ex post measurement of risk, we call this the observed risk.

We now compute the trading gains and losses for the bank. The change in the bank's equity due to fluctuations in the price of the risky asset at time $t+1$ is $\Delta E_{\mathrm{B}}(t)=n(t) \Delta p(t)$, where $\Delta p(t)=p(t+1)-p(t)$. We then define the equity return as

$\ell(t)=\log \left(\frac{E_{\mathrm{B}}(t)+\Delta E_{\mathrm{B}}(t)}{E_{\mathrm{B}}(t)}\right)$.

Note that this captures both the leverage of the bank and the market return of the risky asset since

$\frac{\Delta E_{\mathrm{B}}(t)}{E_{\mathrm{B}}(t)}=\frac{n(t) p(t)}{E_{\mathrm{B}}(t)} \frac{\Delta p(t)}{p(t)}=\lambda(t) w_{\mathrm{B}} r(t)$,

where $r(t)$ is the market return on the risky asset. As expected, leverage amplifies the gains and losses. The total change in equity includes readjustments toward the equity target, but in fact these are small, and the trading losses above are well approximated by the changes in the equity of the bank between time steps. For consistency with standard risk estimation we use log-returns rather than the simple return $r(t)$.

This then leaves us with the question of how to measure risk. To do this we follow current thinking as reflected in Basel III and use realized shortfall. The realized shortfall measures the average tail loss of the bank equity beyond a given quantile $q$. This is the analog to expected shortfall as used in Basel III, except in this case it is based on the profits and losses realized ex post in the simulation of the model. It is a measure of the average loss induced by large market crashes, as shown in Fig. 10. The choice of risk metric is not

\footnotetext{
22 There may be circumstances where the real economy might overheat as a result of too much credit. Nonetheless, we assume that, at a given level of risk, all else equal, the option of being able to obtain more credit is desirable for both borrowers and lenders. When this is not the case they can simply abstain from giving or receiving credit, in which case risk will automatically be lower.
} 
important for the results presented here - we would get similar results with any other reasonable measure of tail risk.

For each set of parameters we estimate the realized shortfall using a time average with $T=5000$ time steps by empirically computing the average loss over the worst $q T$ time observations. ${ }^{23}$ Let $\Theta$ be the indicator function with $\Theta(x)=1$ if $x>0$ and zero otherwise, and let $\ell_{q}$ be the threshold loss corresponding to quantile $0<q<1$, defined through the relation $\sum_{t=1}^{T} \Theta\left(\ell_{q}-\ell(t)\right)=q T$. The realized shortfall at a confidence level $q$ over a time horizon $T$ is defined as

$\mathrm{RS}_{q}=-\frac{1}{q T} \sum_{t=1}^{T} \ell(t) \Theta\left(\ell_{q}-\ell(t)\right)$.

\subsection{Balancing microprudential and macroprudential regulation}

To illustrate how the optimal tradeoff between microprudential and macroprudential regulation depends on the properties of the financial system, in this section we investigate three representative scenarios. The two key properties characterizing the scenarios are the strength of the exogenous clustered volatility and the market impact of the banking sector. The market impact of the banking sector is determined by the product of the average relative size $\hat{R}$ of the banking sector, the average leverage $\hat{\lambda}$ and the bank's portfolio weight $w_{\mathrm{B}}$ for the risky asset. For convenience, to vary the market impact of the banking sector we hold $\hat{\lambda}$ and $w_{\mathrm{B}}$ constant and vary $\hat{R}$.

In each scenario we sweep the cyclicality parameter $b$ in Eq. (2). This determines the degree of procyclicality or countercyclicality of the leverage control policy. As we do this we hold the average leverage and the relative size of the banking sector constant at the stated targets, adjusting $\alpha$ and $\bar{E}$ as needed in order to maintain these targets. We hold all the other parameters of the system constant. ${ }^{24}$ We then measure the observed risk as a function of $b$ and look for a minimum, corresponding to the optimal policy. The results are shown in Fig. 11. below:

We investigate three scenarios, with the results described

1 Microprudential risk dominates (Green diamonds). This occurs when there is strong exogenous clustered volatility and weak bank market impact. To illustrate this we set the GARCH parameters for strong clustered volatility $\left(a_{0}=0.001, a_{1}=0.04, b_{1}=0.95\right)$ and make the banking sector small $\left(\hat{R}=10^{-5}\right)$. In this case there is essentially no systemic risk. The dynamics are dominated by the exogenous volatility, which the historical volatility estimator does a good job of predicting. Not surprisingly, the best leverage control policy is very close to $b=-0.5$, i.e. it corresponds to Basel II. ${ }^{25}$

2 Compromise between microprudential and macroprudential risk (Red squares). This occurs when there is weaker exogenous clustered volatility and intermediate bank market impact. To illustrate this we set the GARCH parameters for weaker clustered volatility $\left(a_{0}=0.001, a_{1}=0.016, b_{1}=0.874\right)$ and increase the

\footnotetext{
${ }^{23}$ For convenience we choose $q$ and $T$ so that their product is an integer.

24 Because the instantaneous leverage $\bar{\lambda}(t)$ and the relative size of the banking sector $R(t)$ are emergent properties that vary in time when there is a leverage cycle, controlling them requires some care. For a given choice of cyclicality parameter $b$ we vary $\alpha$ and $\bar{E}$ to match a target for the average size of the banking sector, $\hat{R}=(1 / T) \sum_{t=0}^{T} R(t)$, and the average leverage $\hat{\lambda}=(1 / T) \sum_{t=0}^{T} \bar{\lambda}(t)$. The leverage is held constant at $\hat{\lambda}=5.8$ for all scenarios but the size of the banking sector $\hat{R}$ varies as stated. All other parameters are as in Table 1 unless otherwise noted.

25 Note that observations for this scenario only extend up to $b \approx 0.1$ as for larger values of $b$ there exists no model solution with the required output targets for relative
} size and leverage.

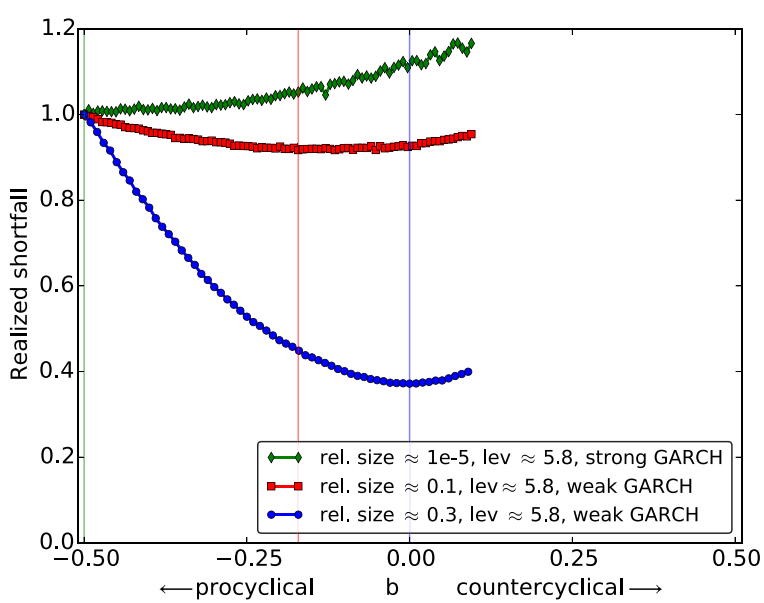

Fig. 11. An illustration of how the proper balance between micro and macroprudential regulation depends on the stability of the financial system. We plot the observed risk as a function of the cyclicality parameter $b$, investigating three different scenarios corresponding to different levels of clustered volatility and different sizes of the banking sector, with other parameters held constant. Realized shortfall has been normalized by $R S_{q}(b=-0.5)$ for ease of comparison. Green diamonds correspond to the case where microprudential risk dominates, i.e. the banking system is relatively small and the exogenous volatility clustering is high. Not surprisingly, the best policy is $b^{*}=-0.5$, i.e. Basel II and is strongly procyclical. Red squares correspond to a mixture of microprudential and macroprudential risk; the size of the banking sector is increased to $\hat{R}=0.1$, and the best policy now has $b^{*} \approx-0.2$, i.e. it is only mildly procyclical. Blue circles correspond to the case where macroprudential risk dominates; the size of the banking sector is increased still further to $\hat{R}=0.27$, and the optimal policy has $b^{*} \approx 0$, i.e. it is very close to constant leverage. See text for parameters. (For interpretation of the references to color in this figure legend, the reader is referred to the web version of this article.)

relative size of the banking sector to $\hat{R}=0.1$. The larger size of the banking sector makes the financial system more prone to endogenous oscillations and the risk is minimized for $b^{*} \approx 0.2$. This corresponds to a leverage control policy that is still procyclical but is nonetheless closer to constant leverage.

3 Macroprudential risk dominates (Blue circles). This occurs when there is weaker exogenous clustered volatility and strong bank market impact. To illustrate this we set the GARCH parameters as in the previous case but increase the relative size of the banking sector still further to $\hat{R}=0.27$. In this case the system becomes very prone to endogenous oscillations and the risk is minimized for $b^{*} \approx 0$, i.e. using a leverage control policy that is very close to constant leverage.

These three scenarios show that the key determinant of the degree to which micro vs. macroprudential regulation is required is the market impact of the banking sector. As this increases the system becomes more prone to oscillation and therefore more susceptible to systemic risk. The dynamics emerge because of the tension between the stabilizing influence of the fundamentalist and the destabilizing influence of the banking sector. As the latter increases in market impact a higher degree of macroprudential regulation is required.

The balance between micro and macroprudential risk can be stated in simple terms as a competition between exogenous vs. endogenously generated volatility. Increasing the size of the banking sector increases the endogenous volatility and means that the system requires a higher level of macroprudential regulation. This is obvious in the model, but of course in the real world it is hard to tell who is generating volatility and therefore difficult to distinguish the two. Nonetheless, the market impact of the banking sector can potentially be estimated by regulators and provides an important systemic risk indicator. 


\section{Conclusion}

\subsection{Summary}

In this paper we have investigated the effect of risk-based leverage policies on financial stability, extending the dynamical model of leverage cycles introduced by Aymanns and Farmer (2015), and roughly calibrating it to match basic features of the S\&P500 trajectory prior and following the $2007 / 2008$ crash.

We considered in particular the effect of different leverage control policies ranging between procyclical and countercyclical ones. Under a procyclical policy the bank decreases its leverage when perceived risk is high. In contrast, under a countercyclical policy the bank is allowed to increase its leverage when perceived risk is high. We study the stability of the model for different values of bank leverage and the cyclicality of the leverage policy. We find three different regimes: (1) For low leverage the system is stable and settles into a fixed point. (2) As leverage is increased leverage cycles emerge. (3) As leverage is increased further the system becomes globally unstable. Our main contribution is the evaluation of different leverage control policies, and our result that the optimal policy depends on the parameters of the financial system: In the microprudential limit when the bank is small and exogenous volatility is high, the optimal policy is simply given by Basel II, i.e. Value-at-Risk $(b=-0.5)$. As the banking sector becomes larger (either through increasing equity or leverage) the optimal policy becomes less procyclical. In the limit when the bank is very large or highly leveraged, the optimal policy is constant leverage, $b=0$.

Our paper clearly illustrates the interplay between exogenous and endogenous volatility: the microprudential response to exogenous volatility can itself cause endogenous volatility which may dominate over exogenous volatility. This insight is crucial for the effective design of macroprudential policies. Such policies must critically evaluate systemic risks, and make an appropriate tradeoff between micro and macroprudential risk.

The results of Section 4.3 give a clear prescription for improved risk management. We show there that lowering the adjustment speed for leverage targets exerts a strong stabilizing force and can have a dramatic effect on the critical leverage.

\subsection{Are our assumptions of bounded rationality reasonable?}

The most interesting aspect of our model is the spontaneous emergence of a leverage cycle, resembling the Great Financial Moderation and the subsequent crisis, and persisting even in the deterministic limit. This suggests that the use of Value-at-Risk, as recommended by Basel II, might have partially caused these events. Of course there are many other possible causes and the real situation is complicated. Nonetheless, our model indicates that the Basel II rules, when combined with aggressive risk taking and the widely use practice of estimating volatility using historically-based moving averages, were sufficient to have caused the Great Financial Moderation and subsequent crisis by themselves.

This model has been criticized for its assumption of bounded rationality. Should not intelligent investors recognize the simple pattern of booms and busts and alter their behavior accordingly?

Perhaps the strongest rebuttal of this criticism is the contradiction with historical facts. As already mentioned there were several prescient warnings about the potential problems with Value-atRisk, but these were largely ignored. Instead some of the greatest minds in economics declared otherwise: In 2003 Robert Lucas said that "the central problem of depression prevention has been solved", in 2004 Ben Bernanke said "improvements in monetary policy, though certainly not the only factor, have probably been an important source of the Great Moderation", and even in 2008
Olivier Blanchard declared that "the state of macro is good", not realizing the possible dangers from financial markets. Our model did not exist then, and the warnings from the models that did exist prior to the crash were not quantitative. Thus it seems that in general neither investors nor regulators were able to anticipate this dynamics.

There are reasons why this was difficult even for intelligent investors. The timescale of the cycle is long - a decade or more - and investors typically lack incentives to anticipate events that in the far future. There are big incentives to participate in bubbles and the timing of crashes is hard to predict. Evolutionary pressure forces funds to take ever-increasing leverage in order to stay competitive, as for example in the model of Thurner et al. (2010). It is difficult for individual investors to fully understand systemic risks based on the incomplete information that is available.

This is made even harder by the fact that these are recent innovations. Value-at-Risk did not become widely used until the mid 1990's and Basel II was adopted in 2004. But perhaps there is hope to do better in the future. Basel III contains countercyclical buffers that are intended to damp leverage cycles. The reliability of this could be tested by constructing a more realistic version of our model. Among other things, this might include allowing the bank's portfolio weights to vary, allowing for phenomena like flight to quality; allowing the possibly of default; modeling the heterogeneity of the financial system; and implementing a more realistic version of the Basel III rules, including risk weights, an asset price-dependent capital buffer and counter cyclicality with respect to macro-economic indicators. Such a model could be developed, and we believe it could provide a useful tool for testing the effectiveness of Basel III and evaluating possible alternatives. One can hope that investors and regulators in the future will be sufficiently rational that we do not have to make the same mistake twice.

\section{Acknowledgements}

This work was supported by the European Union Seventh Framework Programme FP7/2007-2013 under Grant agreement CRISIS-ICT-2011-288501 and by the Institute of New Economic Thinking at the Oxford Martin School. CA has also been supported by the German National Merit Foundation and EPSRC (UK). FC and CA acknowledges support of the Economic and Social Research Council (ESRC) in funding the Systemic Risk Centre (ES/K002309/1). We would like to thank Tobias Adrian and Nina Boyarchenko for useful discussions and Christian Upper for a valuable critique. The code for the model used in this paper is available at https://github. com/ChristophAy/leverage_cycles.

\section{Appendix A. Detailed description of the model}

The model is set in discrete time indexed by $t=\{\tau, 2 \tau, \ldots, T \tau\}$, where $\tau$ is the length of a time-step.

\section{A.1. Assets}

Let $p(t)$ be the price of the risky asset at time $t$. We assume that there is exactly one unit of the risky asset which is infinitely divisible. The return on the price of the risky asset is $r(t)=\log [p(t) / p(t-\tau)]$. The fraction of the risky asset held by the bank is $n(t) \in[0,1]$. Since only the bank and the fund can invest in the risky asset, the fraction of the risky asset held by the fund is simply $1-n(t)$. The risk free asset is analogous to cash. The price of the risk free asset is constant and equal to one. 


\section{A.2. Agents}

There are two representative agents. The first is a bank, denoted $\mathrm{B}$, and the second is a fund, denoted $\mathrm{F}$.

\section{A.3. Bank}

Balance sheet. Assume the bank divides its assets $A_{\mathrm{B}}(t)$ in a fixed ratio $w_{\mathrm{B}}$ between the risky asset and cash $c_{\mathrm{B}}(t)$, so that the banks owns $n(t)$ shares of the risky asset with price $p(t)$. The relevant accounting relations are:

Risky investment $=n(t) p(t)=w_{\mathrm{B}} A_{\mathrm{B}}(t)$,

Risk free investment $=c_{\mathrm{B}}(t)=\left(1-w_{\mathrm{B}}\right) A_{\mathrm{B}}(t)$,

Total assets $=A_{\mathrm{B}}(t)=c_{\mathrm{B}}(t)+n(t) p(t)$.

The bank's liabilities $L_{\mathrm{B}}$ have a maturity of one time step and are freely rolled over or expanded. There is no limit to the reduction in $L_{\mathrm{B}}$; in principle the bank could pay back its entire liabilities in one time step.

The bank adjusts its equity toward a fixed target $\bar{E}$. This guarantees that neither the bank nor the fund asymptotically accumulates all the wealth and makes the long-term dynamics stationary, with only a small effect on the short term dynamics. The dividends paid out by the bank are invested in the fund and new capital invested in the bank comes from the fund. If the bank deviates from its equity target $\bar{E}$ it either pays out dividends or attracts new capital from outside investors at a rate $\eta$ to adjust its equity closer to the target, so that its equity changes by

$\kappa_{\mathrm{B}}(t)=\eta\left(\bar{E}-E_{\mathrm{B}}(t)\right)$.

Taking both the changes in price and the active adjustments in equity into account, the bank's equity at time $t+\tau$ is

$E_{\mathrm{B}}(t+\tau)=n(t) p(t+\tau)+c_{\mathrm{B}}(t)-L_{\mathrm{B}}(t)+\kappa_{\mathrm{B}}(t)$,

and the bank's leverage is

$\lambda(t+\tau)=\frac{\text { Total Assets }}{\text { Equity }}=\frac{n(t) p(t+\tau) / w_{\mathrm{B}}}{E_{\mathrm{B}}(t+\tau)}$.

We assume the bank enforces leverage control through a target leverage $\bar{\lambda}(t)$, corresponding to a target portfolio value $\bar{A}_{\mathrm{B}}(t)(t)=$ $\bar{\lambda}(t) E_{\mathrm{B}}(t)$.

Estimation of perceived risk. The bank relies on historical data to estimate the perceived variance of the risky asset $\sigma^{2}(t)$. To do so the bank computes an exponential moving average of squared returns of the risky asset. This approach is similar to the RiskMetrics approach, see Longerstaey (1996). In particular

$$
\begin{aligned}
\sigma^{2}(t+\tau) & =(1-\tau \delta) \sigma^{2}(t)+\tau \delta r^{2}(t) \\
& =(1-\tau \delta) \sigma^{2}(t)+\tau \delta\left(\log \left[\frac{p(t)}{p(t-\tau)}\right] \frac{t_{\mathrm{VaR}}}{\tau}\right)^{2},
\end{aligned}
$$

where the term $t_{\mathrm{Var}} / \tau$ rescales the return over one time-step $\tau$ to the return over the horizon $t_{\mathrm{VaR}}$ used in the computation of the capital requirement. The parameter $\tau \delta \in(0,1)$ implicitly defines the length of the time window over which the historical estimation is performed. We define the typical time $t_{\delta}$ as the time at which an observation made at $t-t_{\delta}$ has decayed to $1 / e$ of its original contribution to the exponential moving average. Thus $t_{\delta}=-\tau / \log [1-\tau \delta] \approx 1 / \delta$ for $\tau \delta \ll 1$.

\section{A.4. Fund investor}

The fund investor represents the rest of the financial system and plays the role of a fundamentalist noise trader. Since the fund is not leveraged its assets $A_{\mathrm{F}}(t)$ are equal to its equity, i.e. $E_{\mathrm{F}}(t)=A_{\mathrm{F}}(t)$.
Just as for the bank, the fund invests $w_{\mathrm{F}}(t)$ of its assets in the risky asset and $1-w_{\mathrm{F}}(t)$ in cash; a key difference is that the fund adjusts its portfolio weight $w_{\mathrm{F}}(t)$ whereas the bank's weight is fixed. The relevant accounting relations are

Risky investment $=(1-n(t)) p(t)=w_{\mathrm{F}}(t) A_{\mathrm{F}}(t)$,

Risk free investment $=c_{\mathrm{F}}(t)=\left(1-w_{\mathrm{F}}(t)\right) A_{\mathrm{F}}(t)$,

Total assets $=A_{\mathrm{F}}(t)=c_{\mathrm{F}}(t)+(1-n(t)) p(t)$,

and the fund's equity is

$E_{\mathrm{F}}(t+\tau)=(1-n(t)) p(t+\tau)+c_{\mathrm{F}}(t)+\kappa_{\mathrm{F}}(t)$.

The fund's cash flow $\kappa_{\mathrm{F}}:=-\kappa_{\mathrm{B}}$ mirrors the dividend payments or capital injections of the bank.

We have already explained the motivation for the fund's demand function in the main text. Here, we simply note that we rescale the deviation of the price of the risky asset to the fundamental by the current price of the risky asset in order to make portfolio weight adjustments independent of the scale of the price of the risky asset. Otherwise, the portfolio weight would likely exceed its natural bounds, i.e. $w_{\mathrm{F}} \in[0,1]$. In order to introduce heteroskedasticity we make $s^{2}$ time varying according to a simple $\operatorname{GARCH}(1,1)$ process of the form

$$
\begin{aligned}
s^{2}(t) & =a_{0}+a_{1} \chi^{2}(t-1)+b_{1} s^{2}(t-1), \\
\chi(t) & =s(t) \xi(t) .
\end{aligned}
$$

When the parameters $a_{1}$ and $b_{1}$ are zero the returns $r(t)$ of the risky asset are normally distributed as $n(t) \rightarrow 0$, the price process is a mean reverting random walk with constant volatility (i.e. an Ornstein-Uhlenbeck process).

\section{A.5. Market mechanism}

The price of the risky asset is determined by market clearing. For this we construct the demand functions for the bank and fund ( $D_{\mathrm{B}}$ and $D_{\mathrm{F}}$ respectively) as follows:

$$
\begin{aligned}
D_{\mathrm{B}}(t+\tau) & =\frac{1}{p(t+\tau)} w_{\mathrm{B}} A_{\mathrm{B}}(t+\tau) \\
& =\frac{1}{p(t+\tau)} w_{\mathrm{B}}\left(n(t) p(t+\tau)+c_{\mathrm{B}}(t)+\Delta B(t)\right),
\end{aligned}
$$

$$
\begin{aligned}
D_{\mathrm{F}}(t+\tau) & =\frac{1}{p(t+\tau)} w_{\mathrm{F}}(t+\tau) A_{\mathrm{B}}(t+\tau) \\
& =\frac{1}{p(t+\tau)} w_{\mathrm{F}}(t+\tau)\left((1-n(t)) p(t+\tau)+c_{\mathrm{F}}(t)\right) .
\end{aligned}
$$

Recall that there is a supply of exactly one unit of the risky asset that is infinitely divisible. We can then compute the market clearing price by equating demand and supply $1=D_{\mathrm{B}}(t+\tau)+D_{\mathrm{F}}(t+\tau)$. Solving for the market clearing price we obtain

$p(t+\tau)=\frac{w_{\mathrm{B}}\left(c_{\mathrm{B}}(t)+\Delta B(t)\right)+w_{\mathrm{F}}(t+\tau) c_{\mathrm{F}}(t)}{1-w_{\mathrm{B}} n(t)-w_{\mathrm{F}}(t+\tau)(1-n(t))}$

Given the new price we can compute the fraction of the risky asset owned by the bank as follows:

$n(t+\tau)=\frac{1}{p(t+\tau)} w_{\mathrm{B}}\left(n(t) p(t+\tau)+c_{\mathrm{B}}(t)+\Delta B(t)\right)$. 


\section{A.6. Finding the fixed point}

We begin by considering the conditions for a fixed point of the $g(\cdot)$ as defined in Eq. (5). ${ }^{26}$

1 The price is at the noise trader's fundamental value:

$$
p^{*}=\mu \quad \Rightarrow \quad w_{\mathrm{F}}(t+\tau)=w_{\mathrm{F}}(t)
$$

2 The bank's perceived risk is 0 :

$$
\sigma^{2 *}=0 \quad \vee \quad p(t)=p(t-\tau)=\mu \quad \Rightarrow \quad \sigma^{2}(t)=\sigma^{2}(t+\tau)
$$

3 The bank is at its target leverage consistent with $\sigma^{2^{*}}=0$ :

$$
\lambda^{*}=\frac{A_{\mathrm{B}}^{*}}{A_{\mathrm{B}}^{*}-L_{\mathrm{B}}^{*}}=\bar{\lambda}(t)=\alpha\left(\sigma_{0}^{2}\right)^{b} \Rightarrow \Delta B(t)=0
$$

4 The bank is at its target equity:

$$
E_{\mathrm{B}}^{*}=A_{\mathrm{B}}^{*}-L_{\mathrm{B}}^{*}=\bar{E}=\Rightarrow \Delta E_{\mathrm{B}}(t)=0 .
$$

5 The bank's ownership of the risky asset is consistent with the price, leverage target and equity target at the fixed point:

$$
n^{*}=\lambda^{*} E_{\mathrm{B}}^{*} w_{\mathrm{B}} / \mu
$$

The fixed point is therefore:

$$
\begin{aligned}
x^{*} & =\left(\sigma^{2 *}, w_{\mathrm{F}}^{*}, p^{*}, n^{*}, L_{\mathrm{B}}^{*}, p^{\prime *}\right) \\
& =\left(0, w_{\mathrm{F}}(0), \mu, \frac{1}{\mu} \alpha \sigma_{0}^{2 b} \bar{E} w_{\mathrm{B}},\left(\alpha \sigma_{0}^{2 b}-1\right) \bar{E}, \mu\right),
\end{aligned}
$$

where we picked $w_{\mathrm{F}}^{*}=w_{\mathrm{F}}(t=0)$, the initial value of the fund's investment weight, since at $p^{*}=\mu$ any $w_{\mathrm{F}}$ will remain unchanged. Since $w_{\mathrm{F}}^{*}$ is not specified by the fixed point condition, there is essentially a set of fixed points for $w_{\mathrm{F}} \in[0,1]$. As such it is useful to interpret $w_{\mathrm{F}}^{*}$ as a parameter of the model determined by an appropriate initial condition. We choose $w_{\mathrm{F}}(0)=0.5$ throughout.

In the case studies in Figs. 4 and 5 in Section 3 we saw that the properties of the system dynamics depended heavily on the relative proportions of the fund vs. the bank as this determines the impact of the bank on the price of the risky asset. Therefore, before moving on we define the relative size of the bank to the fund at the fixed point as:

$$
R\left(x^{*}\right)=\frac{A_{\mathrm{B}}^{*}}{A_{\mathrm{F}}^{*}}=\frac{\lambda^{*} E_{\mathrm{B}}^{*}}{\left(1-n^{*}\right) p^{*} / w_{\mathrm{F}}^{*}}=\left(\frac{\mu}{\bar{E}} \frac{1}{\alpha \sigma_{0}^{2 b} w_{\mathrm{F}}(0)}-\frac{w_{\mathrm{B}}}{w_{\mathrm{F}}(0)}\right)^{-1} .
$$

Clearly, as the equity of the bank goes up, its size relative to the noise trader will increase. Similarly if the bank risk parameter $\alpha$ or the risk off set $\sigma_{0}^{2}$ is increased, the bank's leverage at the fixed point will increase whereby its size relative to the fund will increase.

\footnotetext{
${ }^{26}$ For the deterministic system it is simple to derive a set of differential equations for the continuous-time limit. We have checked that the qualitative behavior of the system in continuous time is the same as that of the discrete system in this case. For simplicity, and for consistency with Section 5 where numerical simulations for the discrete stochastic case are considered, we present here results for the discrete dynamical system.
}

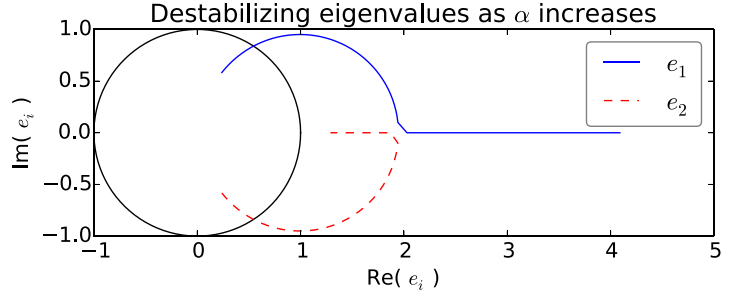

Fig. A.12. Numerical evaluation of the destabilizing eigenvalues (specific results will depend on parameter choice) for different values of $\alpha$. The destabilizing eigenvalues are the two largest eigenvalues that first cross the unit circle from within.

\section{A.7. Existence of critical leverage and bank riskiness}

In order to assess the stability of the fixed point we compute the Jacobian matrix $J_{i j}=\partial g_{i} / \partial x_{j}$. We then evaluate the Jacobian at the fixed point $x^{*}$ and compute the eigenvalues $e_{i}$ of the corresponding matrix. In this particular case the eigenvalues cannot be found analytically. Instead, we compute the eigenvalues numerically using the parameters specified in Table 1. With the help of the eigenvalues we can distinguish between local stability and instability of the fixed point. If the absolute value of the largest eigenvalue $\left|e_{+}\right|>1$ the system exhibits chaotic oscillations, while it is locally stable if $\left|e_{+}\right|<1$. We assess the global stability of the system via numerical iteration of the map in Eq. (5).

Now, suppose we increase the bank risk parameter $\alpha$ and study how the eigenvalues of the Jacobian change while keeping all other model parameters constant. We summarize the evolution of the two largest eigenvalues of the Jacobian in the complex plane in Fig. A.12. The eigenvalues start out at a point within the unit circle on the complex plane (i.e. $\left|e_{i}\right|<1$ ). Then as $\alpha$ is increased the magnitude of the eigenvalues increases. The critical bank riskiness $\alpha_{c}$ at which the eigenvalues cross the unit circle, corresponds to the point at which leverage cycles emerge. Since we keep all other parameters constant, this critical bank riskiness also corresponds to a critical leverage and a critical relative size of the bank to the fund. In particular

$$
\begin{aligned}
& \lambda_{c}^{*}=\alpha_{c} \sigma_{0}^{2 b}, \\
& R_{c}\left(x^{*}\right)=\frac{\lambda_{c}^{*} E_{\mathrm{B}}^{*}}{\left(1-n^{*}\right) p^{*} / w_{\mathrm{F}}^{*}} .
\end{aligned}
$$

\section{References}

Admati, A., Hellwig, M., 2014. The Bankers' New Clothes: What's Wrong with Banking and What to Do about It. Princeton University Press.

Adrian, T., Boyarchenko, N., 2012. Intermediary leverage cycles and financial stability. Staff Reports 567. Federal Reserve Bank of New York.

Adrian, T., Boyarchenko, N., 2012. Intermediary Leverage Cycles and Financial Stability.

Adrian, T., Boyarchenko, N., 2013. Intermediary balance sheets. Staff Reports 651 Federal Reserve Bank of New York, URL: http://ideas.repec.org/p/fip/fednsr/651. html.

Adrian, T., Colla, P., Shin, H., 2012. Which Financial Frictions? Parsing the Evidence from the Financial Crisis of 2007-09. Technical Report. Federal Reserve Bank of New York.

Adrian, T., Shin, H.S., 2008. Liquidity and financial contagion. Finan. Stab. Rev., 1-7. Adrian, T., Shin, H.S., 2010. Liquidity and leverage. J. Finan. Intermed. 19 (3), 418-437. Adrian, T., Shin, H.S., 2014. Procyclical leverage and value-at-risk. Rev. Finan. Stud., 373-403.

Aikman, D., Nelson, B., Tanaka, M., 2015. Reputation, risk-taking, and macroprudential policy. J. Banking Finance 50, 428-439.

Aymanns, C., Farmer, J.D., 2015. The dynamics of the leverage cycle. J. Econ. Dyn. Control 50, 155-179.

Black, F., 1976. Studies of stock price volatility changes. In: Proceedings of the 1976 Proceedings of the Business and Economics Section of the American Statistical Association 10, pp. 171-181.

Brummitt, C.D., Sethi, R., Watts, D.J., 2014. Inside money, procyclical leverage, and banking catastrophes. PLOS ONE 9, e104219.

Brunnermeier, M.K., Pedersen, L.H., 2008. Market liquidity and funding liquidity Rev. Finan. Stud. 22, 2201-2238. 
Christie, A.A., 1982. The stochastic behavior of common stock variances: value, leverage and interest rate effects. J. Finan. Econ. 10, 407-432.

Corsi, F., Marmi, S., Lillo, F., 2013. When Micro Prudence Increases Macro Risk: The Destabilizing Effects of Financial Innovation, Leverage, and Diversification. Technical Report, preprint, http://ssrn.com/abstract=2278298.

Crutchfield, J., Farmer, J., Huberman, B., 1982. Fluctuations and simple chaotic dynamics. Phys. Rep. 92, 45-82.

Danielsson, J., Embrechts, P., Goodhart, C.A.E., Keating, C., Muennich, F., Renault, O., Shin, H.S., 2001. An Academic Response to Basel II.

Danielsson, J., Shin, H.S., Zigrand, J.P., 2004. The impact of risk regulation on price dynamics. J. Banking Finance 28, 1069-1087.

Drehmann, M., Gambacorta, L., 2012. The effects of countercyclical capital buffers on bank lending. Appl. Econ. Lett. 19, 603-608.

Engle, R.F., Ng, V.K., 1993. Measuring and testing the impact of news on volatility. J. Finance 48, 1749-1778.

Estrella, A., 2004. The cyclical behavior of optimal bank capital. J. Banking Finance $28,1469-1498$

Fostel, A., Geanakoplos, J., 2008. Leverage cycles and the anxious economy. Am. Econ. Rev. 98, 1211-1244.

FPC, 2014. The Financial Policy Committee's Powers to Supplement Capital Requirements. Policy Statement. Bank of England.

Geanakoplos, J., 1997. Promises, promises. In: Arthur, W.B., Durlauf, S.N., Lane, D.H. (Eds.), The Economy as an Evolving Complex System, II. Addison-Wesley, Reading, pp. 285-320.

Geanakoplos, J., 2003. Liquidity, default, and crashes endogenous contracts in general. In: Advances in Economics and Econometrics: Theory and Applications: Eighth World Congress, p. 170.

Geanakoplos, J.,2010. The leverage cycle. In: NBER Macroeconomics Annual 2009, vol. 24. University of Chicago Press, pp. 1-65.

Gennaioli, N., Shleifer, A., Vishny, R., 2012. Neglected risks, financial innovation, and financial fragility. J. Finan. Econ., 6452-6468.

Gennotte, G., Leland, H., 1990. Market liquidity, hedging, and crashes. Am. Econ. Rev. 80, 999-1021.
Gorton, G., Metrick, A., 2010. Haircuts. Technical Report. Federal Reserve Bank of St. Louis Review.

He, Z., Krishnamurthy, A., 2012. A model of capital and crises. Rev. Econ. Stud. 79, 735-777, http://dx.doi.org/10.1093/restud/rdr036.

Van den Heuvel, S.J., 2002. Does bank capital matter for monetary transmission? Econ. Policy Rev. 8, 259-265.

Hommes, C.H., 2006. Heterogeneous agent models in economics and finance. In: Judd, K.L., Tesfatsion, L. (Eds.), Handbook of Computational Economics. Elsevier pp. 1110-1146 (Chapter 23)

Longerstaey, J., 1996. Riskmetrics: Technical Document. Technical Report. J.P. Morgan.

Minsky, H.P., 1992. The Financial Instability Hypothesis. The Jerome Levy Economics Institute Working Paper.

Nelson, D.B., 1991. Conditional heteroskedasticity in asset returns. A new approach. Econometrica 59, 347-370.

de Nicolo, G., Favara, G., Ratnovski, L., 2012. Externalities and Macroprudential Policy. Technical Report. IMF.

Peura, S., Keppo, J., 2006. Optimal bank capital with costly recapitalization. J. Business 79, 2163-2201.

Poledna, S., Thurner, S., Farmer, J.D., Geanakoplos, J., 2013. Leverage-Induced Systemic Risk Under Basel II and Other Credit Risk Policies. arXiv:1301.6114.

Shin, H., 2010. Risk and Liquidity. Clarendon Lectures in Finance Ed. Oxford University Press, Oxford.

Shleifer, A., Vishny, R., 2011. Fire sales in finance and macroeconomics. J. Econ. Perspect. 25, 29-48.

Shleifer, A., Vishny, R.W., 1992. Liquidation values and debt capacity: a market equilibrium approach. J. Finance 47, 1343-1366.

Tasca, P., Battiston, S., 2012. Market Procyclicality and Systemic Risk. Technical Report. TH Zurich.

Thurner, S., Farmer, J.D., Geanakoplos, J., 2010. Leverage causes fat tails and clustered volatility. Quant. Finance 12, 695-707.

Zigrand, J.P., Shin, H.S., Danielsson, J., 2010. Risk Appetite and Endogenous Risk. Technical Report. Financial Markets Group. 